\title{
Photon Hanbury-Brown-Twiss interferometry for noncentral heavy-ion collisions
}

\author{
Evan Frodermann* \\ Department of Physics \& Astronomy, University of Minnesota, Minneapolis, Minnesota 55455, USA \\ and Department of Physics, The Ohio State University, Columbus, Ohio 43210, USA \\ Ulrich Heinz \\ Department of Physics, The Ohio State University, Columbus, Ohio 43210, USA
}

(Received 8 July 2009; published 9 October 2009)

\begin{abstract}
Currently, the only known way to obtain experimental information about the space-time structure of a heavy-ion collision is through two-particle momentum correlations. Azimuthally sensitive Hanbury-Brown-Twiss (HBT) intensity interferometry can complement elliptic flow measurements by constraining the spatial deformation of the source and its time evolution. Performing these measurements on photons allows us to access the fireball evolution at earlier times than with hadrons. Using ideal hydrodynamics to model the space-time evolution of the collision fireball, we explore theoretically various aspects of two-photon intensity interferometry with transverse momenta up to $2 \mathrm{GeV}$, in particular the azimuthal angle dependence of the HBT radii in noncentral collisions. We highlight the dual nature of thermal photon emission, in both central and noncentral collisions, resulting from the superposition of QGP and hadron resonance gas photon production. This signature is present in both the thermal photon source function and the HBT radii extracted from Gaussian fits of the two-photon correlation function.
\end{abstract}

DOI: 10.1103/PhysRevC.80.044903

PACS number(s): 25.75.Cj, 25.75.Gz, 25.75.Ld

\section{INTRODUCTION}

Ideal hydrodynamic calculations [1-7] of heavy-ion collisions at the BNL Relativistic Heavy Ion Collider (RHIC) generate momentum distributions of particles that correctly describe the effects of the collision fireball dynamics on the elliptic flow signature and successfully reproduce the main aspects of the measured momentum spectra for a large number of hadron species [8-15]. The good agreement between theory and experiment of the single-particle momentum distributions leaves, however, the fireball geometry essentially unconstrained. To eliminate the geometric ambiguities, spatiotemporal aspects of the reaction must be explored [16]. The only known way to obtain experimental information on the space-time structure of the particle-emitting source in heavyion collisions is through two-particle momentum correlations [17-19]. The method of two-particle intensity interferometry, originally developed by Hanbury Brown and Twiss (HBT) [20] to measure angular distances of stars and other stellar objects, exploits quantum statistical correlations between particle intensities in two-particle coincidence measurements to access spatial information on the emitting source. Even though the space-time picture of the source extracted from intensity interferometry is necessarily incomplete [16,19,21,22], it yields powerful geometric constraints which supplement the momentum-space information contained in the single-particle spectra.

However, most theoretical calculations of the HBT radii, expressed through variances (Gaussian widths) of the source function, do not correctly reproduce their measured momentum dependence $[16,23]$. Various ways to explain this "HBT puzzle" and possibilities to correct this failure have

*Corresponding author: frodermann@physics.umn.edu been suggested. They include a microscopic treatment of the final freeze-out stage through hadronic rescattering [24], exploration of fluctuations in the initial state [25], different sets of initial conditions leading to stronger longitudinal [26] and transverse [27] hydrodynamic acceleration, as well as the inclusion of viscous effects [28-31] and final state interactions [32-35]. The influence on the characteristic HBT radii of finite resonance decay lengths after thermal freeze-out was explored $[27,36,37]$, and the impact of non-Gaussian features in the two-particle correlation function on the extraction of HBT radii from Gaussian fits to the correlator and their discrepancy from radii extracted through source function variances were studied [38]. Recent work [31] suggests that a comprehensive approach that includes all these effects in a theoretically coherent fashion may be able to simultaneously describe single-hadron spectra and two-hadron correlations, lending support to the claim that hydrodynamic models successfully describe both the dynamics and space-time structure (size, shape, and lifetime) of the collision fireball at the point of hadron emission.

An interesting question that cannot be directly addressed by measuring hadron distributions is the time evolution of the collision fireball. The reason is that hadrons interact strongly and decouple late. The observation of strong hadron elliptic flow [8], i.e., a large momentum anisotropy in the final hadron spectra, has been linked to early thermalization in the fireball and to almost perfect liquid behavior of the quark-gluon plasma (QGP) created in RHIC collisions [39,40]. This has opened the prospect of exploring details of the QGP equation of state and of its conversion to hadrons [15] as well as its transport properties (shear and bulk viscosity) through elliptic flow measurements [41-44]. Since the QGP exists only in the early stage of the collision, a more direct probe of its properties and dynamical evolution is desirable rather than through soft hadron spectra emitted after its decay. 
We here discuss the emission of thermal photons which occurs throughout the fireball evolution, but most strongly during its hottest, earliest stage [45-49]. Photons interact only electromagnetically and thus escape from the collision fireball without rescattering. For this advantage over hadrons, one has to pay with a correspondingly smaller production cross section, which makes direct photon measurements much more difficult than the observation of soft hadrons.

The possibility of using photon elliptic flow measurements to access the early evolution of momentum anisotropies in the expanding QGP fireball was explored in Refs. [50-52]. The growth of these momentum anisotropies is driven by spatial anisotropies of the pressure gradients in the initial hot source created in noncentral heavy-ion collisions. The collective flow response of the QGP to these gradient anisotropies depends on its speed of sound and its viscosity [53,54]. We propose that, similar to the way that photon elliptic flow opens a window on the early evolution in momentum space, two-photon HBT interferometry may help constrain the spatial size and deformed shape of the fireball during this stage. By providing access to both the space-time and momentum-space structure of the source at early times, thermal photons may thus help constrain the validity of hydrodynamic models, the QGP EOS, and its transport properties during the crucial hottest and densest period.

While photons are emitted throughout the evolution of the fireball and thus do not allow for sharp snapshots of the early stage alone, their emission rate depends on a high power of the fireball temperature [45-49] which gives preference to early emission [55-59]. Indeed, we will show that the photon emission function has a distinctly bimodal structure, with two components that reflect emission of photon pairs of given pair momentum from different spatial regions at early times (when radial collective flow is still weak) and at late times (when the flow is strong). Although a single measurement is insufficient to cleanly separate these components, systematic exploration of the dependence of photon HBT radii on magnitude and direction of the photon pair momentum may eventually allow us to do so.

The bimodal structure of the photon source is only one of two sources for strong deviations of the emission function from a simple Gaussian shape. The other is the massless nature of the photon. For two-photon correlations, it is therefore even more important than for hadrons that the HBT radius parameters are computed through a procedure that matches their experimental extraction. We here use a generalization to noncentral collisions of the Gaussian fit technique developed in Refs. [38,60]. Since photons, unlike charged hadrons, do not suffer from final-state Coulomb interactions, this technique is even more appropriate here than it is for hadron correlations.

Our study is exploratory in nature and, as such, lacks many features needed for a quantitative analysis. To simulate the dynamical evolution of the matter created in a heavyion collision, we use AZHYDRO, a $(2+1)$-dimensional code developed by P. Kolb [2,7] for solving the equations of ideal fluid dynamics in two transverse dimensions assuming boost invariance along the longitudinal (beam) direction. We use the same photon emission rates as employed in earlier studies of thermal photon spectra and elliptic flow $[50,61,62]$. However, we exclude hard photons produced before the fireball medium has thermalized in order to focus on thermal processes. We also assume that photons from the post-freeze-out decay of hadronic resonances are created sufficiently late that they do not correlate with thermal photons and therefore do not affect the shape of the two-photon correlation function except at immeasurably small relative momenta. (They contribute to the single-photon spectra and will thus affect the normalization of the two-photon correlator, i.e., the correlation strength; this increases the statistics required for an accurate correlation measurement.) Our equation of state assumes chemical equilibrium in the hadronic phase below the critical temperature $T_{c}$ where the QGP converts to hadrons, and thus it does not correctly reflect its measured [63] nonequilibrium chemical composition. All these deficiencies can be removed in time before two-photon correlation measurements become technically feasible.

\section{TWO-PARTICLE CORRELATORS FROM NONCENTRAL COLLISIONS}

\section{A. Correlation and emission functions}

If the emission function is a perfect Gaussian in space-time, the two-particle correlation function is a perfect Gaussian in relative momentum, and the HBT radii can be directly computed from the rms variances of the emission function [22]. Since, however, real emission functions are seldom Gaussian, the direct comparison of rms variances with the experimentally extracted HBT radii should in general be viewed with suspicion. A more reliable (even if more laborious) approach computes from the emission function the actual correlation function and then performs a Gaussian fit of the latter, using the same fit algorithm as employed in experiment [38].

The two-particle correlation function for identical particles with momenta $p_{a}$ and $p_{b}$ is defined as a ratio between the two-particle coincidence cross section and the product of the two single particle spectra as

$$
C\left(\boldsymbol{p}_{a}, \boldsymbol{p}_{b}\right)=\frac{E_{a} E_{b} \frac{d N}{d^{3} p_{a} d^{3} p_{b}}}{E_{a} \frac{d N}{d^{3} p_{a}} E_{b} \frac{d N}{d^{3} p_{b}}} .
$$

If the particles are emitted independently from the source, $C\left(\boldsymbol{p}_{a}, \boldsymbol{p}_{b}\right)$ can be calculated from the single-particle Wigner phase-space density $S(x, K)$ ("emission function") which describes the probability of emitting a particle from space-time point $x$ with momentum $K$, by folding it with the two-particle relative wave function [16]. In the absence of final state interactions (as is true for photon pairs), this wave function is a plane wave, yielding

$$
\begin{aligned}
E \frac{d N}{d^{3} p} & =\int d^{4} x S(x, p), \\
C(\boldsymbol{q}, \boldsymbol{K}) & =1 \pm \frac{1}{g_{s}} \frac{\left|\int d^{4} x S(x, K) e^{i q \cdot x}\right|^{2}}{\int d^{4} x S\left(x, K+\frac{q}{2}\right) \int d^{4} y S\left(y, K-\frac{q}{2}\right)},
\end{aligned}
$$


where $g_{s}$ is the spin degeneracy of the particles (for photons $g_{s}=2$ ), and the $+(-)$ sign applies for bosons (fermions). From now on, we will only consider the bosonic case.

The correlation function in Eq. (3) depends on the relative momentum between the two particles $\boldsymbol{q}=\boldsymbol{p}_{a}-\boldsymbol{p}_{b}, q_{0}=$ $E_{a}-E_{b}$ and the pair momentum $\boldsymbol{K}=\left(\boldsymbol{p}_{a}+\boldsymbol{p}_{a}\right) / 2, K^{0}=$ $\left(E_{a}+E_{b}\right) / 2$. For the spectrum, Eq. (2), the emission function $S(x, K)$ must be evaluated on-shell $(K \mapsto p)$; but for the correlation function (3), this is not necessary [19,21].

Since the measured momenta $p_{a}, p_{b}$ of the particles in the correlator are on-shell, the four-momenta $q$ and $K$ are necessarily off-shell. For pairs of identical particles, the relative and pair momenta satisfy the orthogonality relation

$$
q^{\mu} K_{\mu}=0 .
$$

With this, the argument of the plane wave becomes $q \cdot x=$ $q^{0} t-\boldsymbol{q} \cdot \boldsymbol{x}$, with $q^{0}=E_{a}-E_{b}=\boldsymbol{\beta} \cdot \boldsymbol{q}$, where $\boldsymbol{\beta}=\boldsymbol{K} / K^{0}=$ $2 \boldsymbol{K} /\left(E_{a}+E_{b}\right)$.

Two approximations are often used to further simplify Eq. (3). The first is the "smoothness approximation," which assumes that the emission function varies slowly over the momentum range where the correlator deviates from unity:

$$
C(\boldsymbol{q}, \boldsymbol{K}) \approx 1+\frac{1}{g_{s}}\left|\frac{\int d^{4} x S(x, K) e^{i q \cdot x}}{\int d^{4} x S(x, K)}\right|^{2} .
$$

It is accurate as long as the curvature of the logarithm of the single-particle spectrum is small [64]. For thermal sources created in heavy-ion collisions, this is usually an excellent approximation [65]. The second is the "on-shell approximation" $K^{0} \equiv\left(E_{a}+E_{b}\right) / 2 \approx E_{K} \equiv \sqrt{m^{2}+\boldsymbol{K}^{2}}$. It allows us to replace the factor $\boldsymbol{\beta}$ in the relative wave function by the pair velocity and to substitute for the Wigner densities in Eq. (5) the classical phase-space distributions for on-shell particles [66]. Writing

$$
\begin{aligned}
K^{0} & =\frac{1}{2}\left(E_{a}+E_{b}\right) \\
& =\frac{E_{K}}{2}\left(\sqrt{1+\frac{q^{2}}{4 E_{K}^{2}}+\frac{\boldsymbol{K} \cdot \boldsymbol{q}}{E_{K}^{2}}}+\sqrt{1+\frac{q^{2}}{4 E_{K}^{2}}-\frac{\boldsymbol{K} \cdot \boldsymbol{q}}{E_{K}^{2}}}\right) \\
& =E_{K}\left[1+\frac{q^{2}}{8 E_{K}^{2}}\left(1-\cos ^{2} \theta_{q K}\right)+\mathcal{O}\left(\frac{q^{4}}{E_{K}^{4}}\right)\right] \approx E_{K},
\end{aligned}
$$

we see that it applies as long as $q /\left(2 E_{K}\right) \ll 1$, i.e., as long as the source radius is much larger than the Compton wavelength of the particle pair [22,65]. In heavy-ion collisions, this holds for all hadron species (including pions) at all pair momenta $\boldsymbol{K}$, due to their large rest masses. For massless photons, on the other hand, the on-shell approximation breaks down at small pair momenta. For $m=0$ and $K \ll q / 2$, one obtains, instead of Eq. (6),

$$
K^{0}=\frac{1}{2}\left[\frac{q}{2}\left(\sqrt{1+\frac{4 K^{2}}{q^{2}}+\frac{4 \boldsymbol{K} \cdot \boldsymbol{q}}{q^{2}}}+\sqrt{1+\frac{4 K^{2}}{q^{2}}-\frac{4 \boldsymbol{K} \cdot \boldsymbol{q}}{q^{2}}}\right)\right]
$$

$$
=\frac{q}{2}\left[1+\frac{2 K^{2}}{q^{2}}\left(1-\cos ^{2} \theta_{q K}\right)+\mathcal{O}\left(\frac{K^{4}}{q^{4}}\right)\right] \approx \frac{q}{2} .
$$

Hence, for small-momentum photon pairs, $\boldsymbol{\beta}=\boldsymbol{K} / K^{0} \approx$ $2 K / q$ (which obviously differs from the pair velocity that has magnitude $c=1$ ). We will see that this has interesting consequences for the structure of the two-photon correlation function and the $K$ dependence of photon HBT radii.

\section{B. Gaussian sources and rms variances}

The main characteristic of the correlation function is the $q$ range over which it decays to 1 . The corresponding halfwidths of the correlator are called "HBT radius parameters" or "HBT radii." For a Gaussian source, they can be related to the "homogeneity lengths" (defined below) of the source [19]. In the absence of final state interactions, they can be extracted by fitting the correlator $C(\boldsymbol{q}, \boldsymbol{K})$ with a Gaussian function of the form

$$
C(\boldsymbol{q}, \boldsymbol{K})=1+\lambda(\boldsymbol{K}) \exp \left[-\sum_{i j=x, y, z} q_{i} q_{j} R_{i j}^{2}(\boldsymbol{K})\right],
$$

where the correlation strength parameter $\lambda(\boldsymbol{K})$ is introduced to account for uncorrelated pairs that contribute to the singleparticle spectra in the denominator but not to the correlated part of the two-particle cross section in the numerator. Uncorrelated pairs arise from far-separated decay products of long-lived resonances and (if the particles carry spin) from pairs with unaligned particle spins (e.g., photons with opposite helicity). The correlation strength is also reduced if particles are not emitted independently, but partially coherently [22].

In general, the HBT radii $R_{i j}^{2}$ depend on the pair momentum. They are interpreted as width parameters of the effective source ("homogeneity regions" [67]) from which particles with momentum $\boldsymbol{K}$ are emitted. In collectively expanding sources, where the particle momenta are correlated with position by a boost with the local flow velocity, the homogeneity regions for particles with a given momentum constitute only a fraction of the entire fireball. However, only the homogeneity lengths can be measured with HBT correlations. Furthermore, the HBT radii measure only certain combinations of spatial and temporal width parameters ("variances") of the source, as imposed by the mass-shell constraint (4), which implies $q \cdot x=-\boldsymbol{q} \cdot(\boldsymbol{x}-\boldsymbol{\beta} t)$ :

$$
\begin{aligned}
R_{i j}^{2}(\boldsymbol{K}) & =\left\langle\left(\tilde{x}_{i}-\beta_{i} \tilde{t}\right)\left(\tilde{x}_{j}-\beta_{j} \tilde{t}\right)\right\rangle \\
& \equiv-\left.\frac{1}{2} \frac{\partial^{2} C(\boldsymbol{q}, \boldsymbol{K})}{\partial q_{i} \partial q_{j}}\right|_{\boldsymbol{q}=0} .
\end{aligned}
$$

Here $\langle f\rangle$ denotes an average over the the emission function,

$$
\langle f\rangle \equiv \frac{\int d^{4} x f(x) S(x, \boldsymbol{K})}{\int d^{4} x S(x, \boldsymbol{K})},
$$

and $\tilde{x}^{\mu} \equiv x^{\mu}-\left\langle x^{\mu}\right\rangle$. The relations (10) are exact for Gaussian sources where the inverse width of the correlator agrees with its curvature at $\boldsymbol{q}=0$. In this case, they can be used as a shortcut for the calculation of HBT radii, by evaluating the rms variances in Eq. (10) directly from the emission function 
$S(x, K)$ instead of first computing the correlator from Eq. (5) and then fitting it with a Gaussian as in Eq. (9). Here we will not use this shortcut but instead show results obtained with the help of Eq. (10) only for comparison purposes, to illustrate the importance of non-Gaussian features in the source and correlators.

\section{Gaussian fitting procedure for noncentral collisions}

The emission function $S(x, K)$ is computed from the hydrodynamic model AZHYDRO, for hadrons as described in Ref. [15] and for photons as outlined in Refs. [50,51,61], which folds the thermal photon emission rate with the hydrodynamic temperature and flow evolution. Using this emission function in the average (11), we compute the correlation function (5) as

$$
g_{s}(C(\boldsymbol{q}, \boldsymbol{K})-1)=\langle\cos (q \cdot x)\rangle^{2}+\langle\sin (q \cdot x)\rangle^{2},
$$

where

$$
q \cdot x=\left(E_{a}-E_{b}\right) t-q_{x} x-q_{y} y-q_{l} z,
$$

with $E_{a, b}^{2}=E_{K}^{2} \pm \boldsymbol{K} \cdot \boldsymbol{q}+\boldsymbol{q}^{2} / 4$. We will only consider "midrapidity pairs" with zero pair momentum along the beam direction $\left(K_{l}=0\right)$ such that $\boldsymbol{K} \cdot \boldsymbol{q}=K_{\perp} q_{o}$, where $q_{o}$ denotes the "outward" component of the relative momentum (along the emission direction of the pair in the transverse plane). The "sideward" component $q_{s}$ is defined as the one perpendicular to $\boldsymbol{K}_{\perp}$ and the beam direction.

For collisions between equal-mass nuclei and pairs emitted with zero longitudinal momentum in the center-of-mass frame, the source is reflection symmetric in the longitudinal direction, and the general form (9) reduces to $[68,69]$

$$
C\left(\boldsymbol{q}, \boldsymbol{K}_{\perp}\right)=1+\lambda e^{-\left(q_{o}^{2} R_{o}^{2}+q_{s}^{2} R_{s}^{2}+q_{l}^{2} R_{l}^{2}+2 q_{o} q_{s} R_{o s}^{2}\right)},
$$

where $\lambda, R_{o}, R_{s}, R_{l}$, and $R_{o s}$ are all dependent on $\boldsymbol{K}_{\perp}$.

For central collisions between spherical nuclei, the emission function is azimuthally symmetric, and all directions of $\boldsymbol{K}_{\perp}$ are equivalent (i.e., the correlator and HBT radii only depend on the magnitude of $K_{\perp}$ ). As a consequence, the cross term $R_{o s}^{2}$ vanishes [68,69]. In noncentral collisions, the source is deformed and initially out-of-plane elongated with respect to the reaction plane spanned by the impact parameter $\boldsymbol{b}$ (defining the $x$ axis) and the beam (defining the $z$ axis). As a result, the cross term $R_{o s}^{2}$ is now nonzero, and the two-particle correlation function (12) and its HBT radii (14) now depend on the azimuthal angle $\Phi$ of the emission direction $\boldsymbol{K}_{\perp}$. This dependence is both implicit through azimuthal symmetry violations in the emission function $S(x, K)$ with which the averages in Eq. (12) are taken and explicit through the azimuthal rotation between the reaction-plane coordinate system (in which the source has been computed) and the $o s l$ system defined by the emission direction of the pair in which the HBT radii in Eq. (14) are determined [68-70]:

$$
\begin{aligned}
& q_{x}=q_{o} \cos \Phi-q_{s} \sin \Phi, \\
& q_{y}=q_{o} \sin \Phi+q_{s} \sin \Phi .
\end{aligned}
$$

The HBT radii are determined by fitting the computed correlation function with the functional form (14). To this end we generalize the Gaussian fitting algorithm developed in
Refs. [38,60] to include the $R_{o s}$ as an additional fit parameter. (Note that $R_{o s}$ cannot be determined from one-dimensional Gaussian fits to slices of the correlation function along any one of the osl axes; it requires at least a two-dimensional Gaussian fit in the os plane.) We write

$$
\begin{aligned}
\ln \left[g_{s}(C(\boldsymbol{q})-1)\right]= & \ln \lambda-\left(q_{o}^{2} R_{o}^{2}+q_{s}^{2} R_{s}^{2}\right. \\
& \left.+q_{l}^{2} R_{l}^{2}+2 q_{o} q_{s} R_{o s}^{2}\right),
\end{aligned}
$$

evaluate this expression on a suitable set of $\boldsymbol{q}$ points $\boldsymbol{q}^{(k)}(k=$ $1, \ldots, N)$, and define $\chi^{2}$ as

$$
\chi^{2}=\sum_{k=1}^{N}\left[\frac{\ln \left[C\left(\boldsymbol{q}^{(k)}\right)-1\right]-\ln \lambda+M\left(\boldsymbol{R}, \boldsymbol{q}^{(k)}\right)}{\sigma_{k}^{\prime}}\right]^{2},
$$

with

$$
M\left(\boldsymbol{R}, \boldsymbol{q}^{(k)}\right)=2 q_{o}^{(k)} q_{s}^{(k)} R_{o s}^{2}+\sum_{i=o, s, l}\left(q_{i}^{(k)}\right)^{2} R_{i}^{2} .
$$

For the bin error $\sigma_{k}^{\prime}$ we use [38]

$$
\sigma_{k}^{\prime}=\frac{\sigma_{k}}{C\left(\boldsymbol{q}^{(k)}\right)-1} .
$$

Minimization of $\chi^{2}$ with respect to the fit parameters,

$$
\frac{\partial \chi^{2}}{\partial \ln \lambda}=0, \quad \frac{\partial \chi^{2}}{\partial R_{i}^{2}}=0 \quad(i=o, s, l, o s),
$$

produces a set of five coupled linear equations that can be written in matrix form as

$$
\sum_{\beta} T_{\alpha \beta} P_{\beta}=V_{\alpha},
$$

where $\alpha$ and $\beta$ take the values $\varnothing, o, s, l, o s$ ( $\varnothing$ is associated with the correlations strength $\lambda$ ). The vectors on the right-hand side have the form

$$
\begin{aligned}
P & =\left(\ln \lambda, R_{o}^{2}, R_{s}^{2}, R_{l}^{2}, R_{o s}^{2}\right), \\
V_{\phi} & =-\sum_{k=1}^{N} \frac{\ln \left[C\left(\boldsymbol{q}^{(k)}\right)-1\right]}{\left(\sigma_{k}^{\prime}\right)^{2}}, \\
V_{i} & =+\sum_{k=1}^{N} \frac{\left(q_{i}^{(k)}\right)^{2}}{\left(\sigma_{k}^{\prime}\right)^{2}} \cdot \ln \left[C\left(\boldsymbol{q}^{(k)}\right)-1\right], \\
V_{o s} & =+2 \sum_{k=1}^{N} \frac{q_{o}^{(k)} q_{s}^{(k)}}{\left(\sigma_{k}^{\prime}\right)^{2}} \cdot \ln \left[C\left(\boldsymbol{q}^{(k)}\right)-1\right],
\end{aligned}
$$

while the symmetric $5 \times 5$ matrix $T$ has the components

$$
\begin{aligned}
T_{\phi \phi} & =-\sum_{k=1}^{N} \frac{1}{\left(\sigma_{k}^{\prime}\right)^{2}}, \\
T_{\phi i} & =+\sum_{k=1}^{N} \frac{\left(q_{i}^{(k)}\right)^{2}}{\left(\sigma_{k}^{\prime}\right)^{2}}, \\
T_{i j} & =-\sum_{k=1}^{N} \frac{\left(q_{i}^{(k)}\right)^{2}\left(q_{j}^{(k)}\right)^{2}}{\left(\sigma_{k}^{\prime}\right)^{2}}, \\
T_{\phi, o s} & =+2 \sum_{k=1}^{N} \frac{q_{o}^{(k)} q_{s}^{(k)}}{\left(\sigma_{k}^{\prime}\right)^{2}},
\end{aligned}
$$




$$
T_{i, o s}=-2 \sum_{k=1}^{N} \frac{\left(q_{i}^{(k)}\right)^{2}\left(q_{o}^{(k)} q_{s}^{(k)}\right)}{\left(\sigma_{k}^{\prime}\right)^{2}},
$$

with $\{i, j\}=(o, s, l)$. As before [38], this set of linear equations is easily solved through matrix diagonalization of $T_{\alpha \beta}$.

\section{TWO-PION CORRELATIONS IN NONCENTRAL $\mathrm{Au}+\mathrm{Au}$ COLLISIONS}

As a reference for comparison with the two-photon correlation functions computed further below, we briefly review the behavior of two-pion correlations and pion HBT radii in noncentral $\mathrm{Au}+\mathrm{Au}$ collisions at RHIC energies. This analysis complements earlier work [71] which was based on the same hydrodynamical model as used here but made use of the shortcut (10) to calculate the HBT radii directly from the rms variances of the hydrodynamic pion emission function. As explained above and numerically studied in Ref. [38], this shortcut becomes doubtful when the emission function is not well described by a Gaussian in space-time. Here, we first compute the two-pion correlator (5) numerically from the hydrodynamic emission function $S_{\pi}(x, K)$ [72] and then obtain the HBT radii from a three-dimensional Gaussian fit to this correlation function as described in the preceding section. For simplicity, we include only directly emitted pions; i.e., we neglect pions from post-freeze-out decays of unstable resonances.

Figure 1 shows the azimuthal angle dependence of the pion correlation function for peripheral $\mathrm{Au}+\mathrm{Au}$ collisions at $b=7 \mathrm{fm}$, in the limit $K_{\perp} \rightarrow 0$, as we change the direction of $\boldsymbol{K}_{\perp}$. At $K_{\perp}=0$, the correlation function "sees" the entire fireball [71]. Correspondingly, for noncentral collisions at RHIC energies, the outward radius increases and the sideward radius decreases as we move away from $\Phi=0$ because of the out-of-plane deformation of the source at freeze-out. Beyond $\Phi=90^{\circ}$ these tendencies reverse. Through geometric arguments, one easily sees that these oscillations are seen as a clockwise rotation of the correlator contours, opposite to the counter-clockwise rotation of the direction of the transverse pair momentum. These same oscillations were observed in

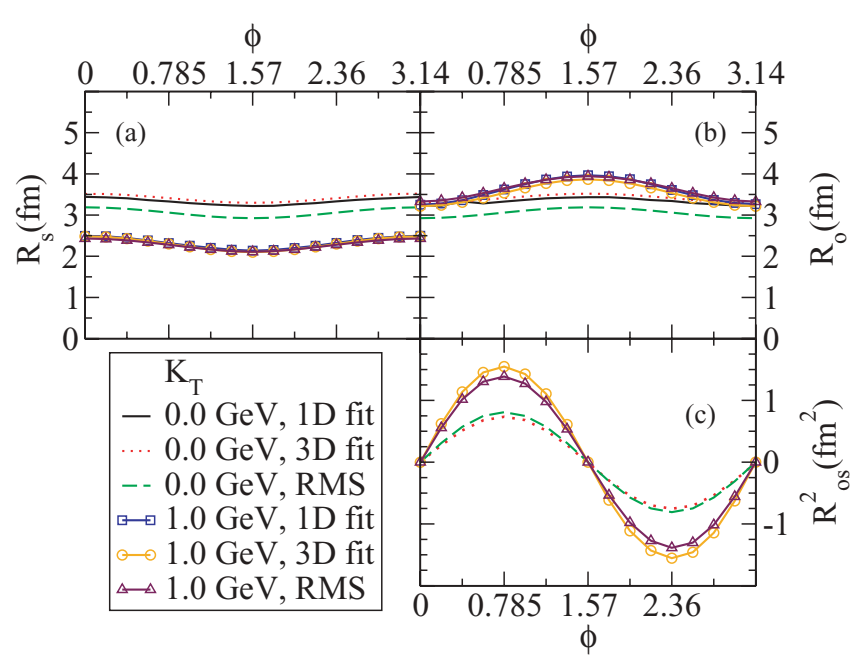

FIG. 2. (Color online) Pion HBT radii $R_{o}, R_{s}$, and $R_{o s}^{2}$ from semiperipheral $(b=7 \mathrm{fm}) \mathrm{Au}+\mathrm{Au}$ oscillations at $\sqrt{s_{N N}}=$ $200 \mathrm{GeV}$, as functions of the azimuthal emission angle $\Phi$. See text for discussion of different extraction methods.

previous calculations that used rms variances of the emission function as proxies for the HBT radii [71].

Figure 2 compares the azimuthal oscillations of the pion HBT radii for $200 A \mathrm{GeV} \mathrm{Au}+\mathrm{Au}$ collisions at $b=7 \mathrm{fm}$ with those of the rms radii, for two values of $K_{\perp}(0$ and $1 \mathrm{GeV} / c)$. In addition to the results from the three-dimensional (3D) Gaussian fit described in the previous section, we also show, for the purpose of comparison, radius parameters extracted from one-dimensional (1D) Gaussian fits to slices of the correlation function along the $q_{s}$ and $q_{o}$ axes. (Note that the $R_{o s}^{2}$ cross term cannot be extracted from these slices, so the corresponding 1D curves are missing.) In the 3D Gaussian fits, we restrict the $q_{l}$ fit range to a narrow window $q_{l}<q_{\max }=0.02 \mathrm{GeV}$, in order to avoid distortions in $R_{o}$ arising from strong non-Gaussian tails of the correlator at larger $q_{l}$ values (see Ref. [38] and Fig. 7 below). This is permissible because we found that the shape of the correlation function along the $q_{l}$ direction is almost independent of $\Phi$, including its non-Gaussian tail. The reason is that even though non-Gaussian effects of the

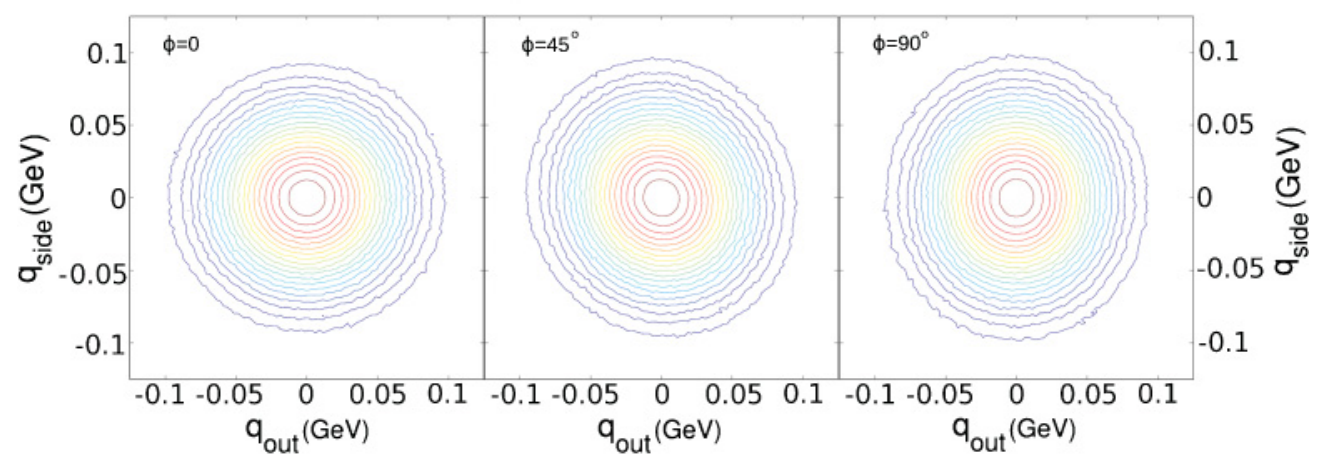

FIG. 1. (Color online) Contour plots of the pion correlation function $C\left(q_{o}, q_{s}, q_{l}=0\right)-1$, at $\Phi=0^{\circ}, 45^{\circ}$, and $90^{\circ}$ in the limit $K_{\perp} \rightarrow 0$, for $200 \mathrm{~A} \mathrm{GeV} \mathrm{Au}+\mathrm{Au}$ collisions at $b=7 \mathrm{fm}$. When rotating $\boldsymbol{K}_{\perp}$ counter-clockwise, the correlation function rotates clockwise. Successive contours are separated by 0.05 , starting from $C(0)-1=1$ in the center. 
source are strongest along the beam direction, they are mainly caused by the strong boost-invariant longitudinal expansion of the fireball, which is independent of impact parameter and transverse position in the reaction zone and thus $\Phi$ independent. If we use a larger fit range in $q_{l}$, thereby capturing more of the non-Gaussian tail, we find an intercept parameter $\lambda<1$ that oscillates with $\Phi$. These oscillations interfere with the oscillations of the transverse radius parameters $R_{o}$ and $R_{S}$, significantly modifying their oscillation amplitudes. This is an undesirable effect caused by non-Gaussian features which lie entirely in the longitudinal domain. By restricting the fit range in the $q_{l}$ direction as described, we can keep the correlation strength parameter close to unity for all angles and ensure that the azimuthal oscillations of the transverse HBT radii faithfully reflect the oscillations in the widths of $C\left(q_{o}, q_{s}\right)$ as seen in the contour plots.

Figure 2 shows that the azimuthal oscillation amplitudes of the HBT radii from the Gaussian fit agree very well with those of the rms radii even though (as previously observed [38]) the $\Phi$-averaged HBT radii differ somewhat from the corresponding rms radii. $R_{o s}^{2}$ is seen to increase with $K_{\perp}$, continuing the previously predicted [71,73] and experimentally confirmed [74] trend to larger values of $K_{T}$.

\section{PHOTON INTERFEROMETRY}

Since photons are emitted throughout the fireball evolution, with emission at higher transverse momenta weighted toward earlier times and higher temperatures, we expect (and our calculations confirm) that for sufficiently high $K_{\perp}$, the thermal photon HBT radii will reflect geometric and dynamical characteristics of the smaller and more deformed early source. This is a simple consequence of the dominance of QGP radiation in the single-photon yield at high $p_{\perp}$. However, similar to pion interferometry, at any given $K_{\perp}$, contributions from lower temperature regions that are blue shifted by collective flow will mix with the early emission contributions. This lower temperature emission is generated at later times when the source is larger and less deformed. There are two competing processes: small radii with weak flow and large source deformations reflected in the early emitted photons and larger radii with smaller spatial deformation which characterizes the emission from the more strongly expanding later stages, superimposed by a nontrivial $K_{\perp}$ dependence driven by longitudinal boost dynamics. The complexity of these competing processes demands a comprehensive study of two-photon interferometry and the corresponding HBT radii.

Bass et al. [59] explored photon interferometry for central $\mathrm{Au}+\mathrm{Au}$ collisions using ideal hydrodynamics coupled to a parton cascade to simulate the early preequilibrium emission [75]. They did not, however, investigate the azimuthal behavior of the photon HBT radii in noncentral collisions. In this work, we pioneer the exploration of anisotropies of the photon HBT radii from noncentral heavy-ion collisions, using the same $(2+1)$-dimensional hydrodynamic source exploited in our earlier work on photon elliptic flow $[50,51,61,62]$ to provide us with the thermal photon emission function. We examine photon HBT radii from both central and noncentral collisions and compare them with the corresponding pion HBT radii.

Before proceeding, we must address several important differences between pion photon interferometry. First, for pions, we were able to ignore spin. Photons carry spin 1 and possess two possible helicity states, and only photons with aligned helicities contribute to Bose-Einstein correlations through wave function symmetrization. Consequently, the strength of the photon correlator is reduced by a factor of 2 [76]. The correlator between two photons with momenta $\boldsymbol{p}_{a}=\boldsymbol{K}+\frac{1}{2} \boldsymbol{q}$ and $\boldsymbol{p}_{b}=\boldsymbol{K}-\frac{1}{2} \boldsymbol{q}$, averaged over helicities, thus reads

$$
C(\boldsymbol{q}, \boldsymbol{K}) \approx 1+\frac{1}{2}\left|\frac{\int d^{4} x S(x, \boldsymbol{K}) e^{i \boldsymbol{q} \cdot \boldsymbol{x}}}{\int d^{4} x S(x, \boldsymbol{K})}\right|^{2},
$$

where we again applied the smoothness approximation, approximating the source functions at $\boldsymbol{K} \pm \frac{1}{2} \boldsymbol{q}$ in the denominator of Eq. (3) by source functions at $\boldsymbol{K}$. A second, less obvious difference between two-pion and two-photon correlations arises from the fact that photons are massless, which is addressed next.

\section{A. Failure of rms variances for soft photons}

It turns out that for soft photon pairs $\left(\left(|\boldsymbol{K}| \lesssim 1 / R_{\text {source }}\right)\right)$, both the on-shell and smoothness approximations discussed in Sec. II A become problematic. In this subsection, we consider these approximations in turn.

\section{On-shell approximation}

Consider the source rms radii $R_{O}$ and $R_{s}$ defined in Eq. (10),

$$
R_{o}^{2}=\left\langle\tilde{x}_{o}^{2}\right\rangle-2 \beta\left\langle\tilde{x}_{o} \tilde{t}\right\rangle+\beta^{2}\left\langle\tilde{t}^{2}\right\rangle, \quad R_{s}^{2}=\left\langle\tilde{x}_{s}^{2}\right\rangle,
$$

where for midrapidity pairs, $\beta=\beta_{\perp}=K_{\perp} / K^{0}$. With the onshell approximation $K^{0}=\left(E_{a}+E_{b}\right) / 2 \approx E_{K}$ (see Sec. II A), this becomes the pair velocity which for massless photons is $\beta=1$, independent of transverse pair momentum. The on-shell approximation is valid for $q \ll 2 K_{\perp}$, so it holds at $\boldsymbol{q}=0$ such that the rms radii (10) and (28) continue to correctly describe the curvature of the correlation function $C(\boldsymbol{q}, \boldsymbol{K})$ at the origin. Equation (28) shows that for sources with nonzero emission duration $\left\langle\tilde{t}^{2}\right\rangle$ the outward and sideward rms radii (and thus the curvature radii at the origin of the correlator in the corresponding $\boldsymbol{q}$ directions) differ from each other at all values of $K_{\perp}$. This is contrary to the case of massive hadrons, where the temporal contributions to the rms radii are suppressed at $K_{\perp} \rightarrow 0$ by powers of the pair velocity $\beta \rightarrow 0$ such that generically $R_{s}\left(K_{\perp}=0\right)=R_{o}\left(K_{\perp}=0\right.$ ) (for a discussion of possible exceptions, see Ref. [77]).

The validity of the on-shell approximation is restricted to the region $q \ll 2 E_{K}$, which for midrapidity photon pairs shrinks to zero as $K_{\perp} \rightarrow 0$. This implies that for $K_{l}=0$ photons with small transverse pair momenta $K_{\perp} \lesssim 1 / R_{\text {source }}$ (where $R_{\text {source }}$ characterizes the size of the emission region), the rms radii (10) describing the curvature of the correlator at $\boldsymbol{q}=0$ can no longer be expected to faithfully represent the width of the correlator. In other words, for photons, the 
standard connection that for Gaussian sources relates the inverse width of the two-photon correlator to the size of the photon-emitting region is broken for small pair momenta. Consequently, for soft photons, the HBT radii extracted from a Gaussian fit to the two-photon correlation function cannot be directly computed from the rms widths of the photon emission function, even if the latter is perfectly Gaussian. At large $K_{\perp} \gg 1 / R_{\text {source }}$, this remains possible for Gaussian photon emission functions. However, since hydrodynamic photon emission functions are not sufficiently Gaussian (see below), the rms shortcut should be avoided altogether and replaced by a Gaussian fit to the numerically computed two-photon correlation function.

We illustrate this issue in Fig. 3 by studying the small- $q$ behavior of the two-photon correlation function for soft midrapidity photon pairs with $K_{\perp}=0.01 \mathrm{GeV} / c$, computed numerically from the hydrodynamic photon emission function. Shown are 1D slices of $C-1$ along the three axes $q_{s}, q_{o}$, and $q_{l}$ (from top to bottom), setting the two other $q$ components to zero. By plotting $C(\boldsymbol{q}, \boldsymbol{K})-1 \operatorname{logarithmically}$ against $q_{i}^{2}$, one easily identifies non-Gaussian features as deviations from linear behavior. The Gaussian width parameters $R_{i}^{2}$ are given by the inverse slopes of the lines. Clearly, $R_{l}^{2}>R_{o}^{2}>R_{s}^{2}$ in the case shown. One sees that $C-1$ is a perfect Gaussian in the sideward but not in the outward and longitudinal directions. Deviations from Gaussian behavior along the $q_{l}$ direction should be expected, since they are also seen in the two-pion correlation functions [38] and can be traced $[64,78]$ to the boost-invariant expansion dynamics of our hydrodynamic source. They extend over the entire $q_{l}$ range, i.e., $\ln \left[C\left(q_{l}^{2}\right)-1\right]$ cannot be approximated by a straight line anywhere (except for $q_{l}=0$ ). This is different for the outward correlator $\ln \left[C\left(q_{o}^{2}\right)-1\right]$ (solid line in Fig. 3), which can be quite well described by two straight lines with different

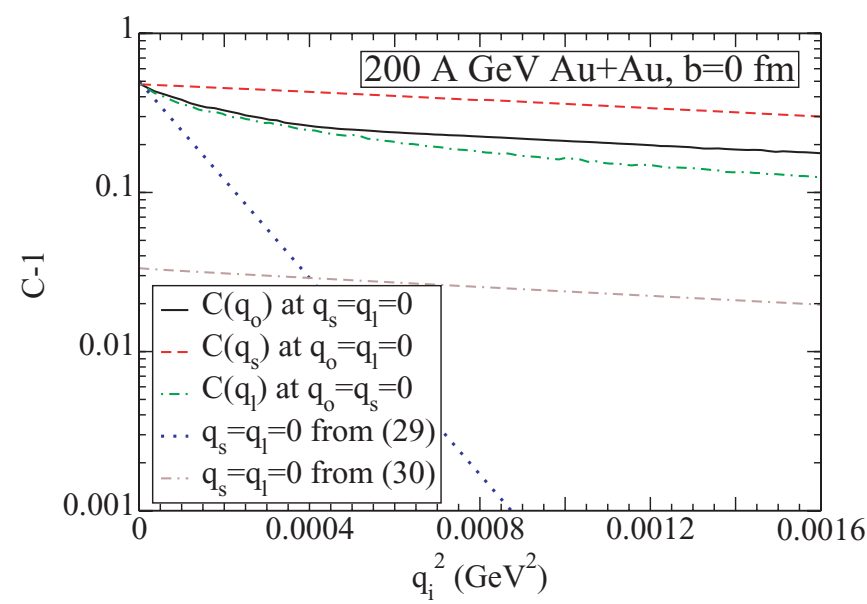

FIG. 3. (Color online) One-dimensional slices of the two-photon correlation function $C(\boldsymbol{q}, \boldsymbol{K})$ for soft photons from central $200 \mathrm{~A} \mathrm{GeV}$ $\mathrm{Au}+\mathrm{Au}$ collisions, for midrapidity pairs with $K_{\perp}=0.01 \mathrm{GeV} / c$. $C-1$ is plotted logarithmically against $q_{i}^{2}(i=o, s, l)$ to facilitate visual extraction of the Gaussian "width parameters" from the slopes of the curves. The blue dotted and brown double-dash-dotted lines are computed from the rms variances of the source via Eqs. (29) and (30), respectively. See text for discussion. slopes at low and high $q_{o}^{2}$, with a smooth transition near $q_{o}^{2}=0.0004(\mathrm{GeV} / c)^{2}=\left(2 K_{\perp}\right)^{2}$. Apparently, this change of slope is associated with the transition between the two limits discussed at the end of Sec. II A. Such a change of slope is not seen for the two-pion correlator, which for our hydrodynamical source is quite well described by a single Gaussian in the outward direction [38].

We can try to understand the behavior of the curves in Fig. 3 analytically by writing (for $K_{l}=0$ pairs) $\beta=K_{\perp} / K^{0}(q) \equiv$ $\beta(q)$, with the limits $\lim _{q \ll 2 K_{\perp}} \beta(q)=1$ and $\lim _{q \gg 2 K_{\perp}} \beta(q)=$ $2 K_{\perp} / q$ for massless photons, and using this to express the exponent in Eq. (9) in terms of the rms variances (10), assuming a Gaussian source. In the limit of small relative momenta $q \ll 2 K_{\perp}$, we obtain

$$
\begin{aligned}
& \sum_{i j} q_{i} q_{j}\left\langle\left(\tilde{x}_{i}-\beta_{i}(q) \tilde{t}\right)\left(\tilde{x}_{j}-\beta_{j}(q) \tilde{t}\right)\right\rangle \\
&=\left\{\begin{array}{cl}
q_{s}^{2}\left\langle\tilde{x}_{s}^{2}\right\rangle, & q_{o}=q_{l}=0, \\
q_{o}^{2}\left(\left\langle\tilde{x}_{o}^{2}\right\rangle-2\left\langle\tilde{x}_{o} \tilde{t}\right\rangle+\left\langle\tilde{t}^{2}\right\rangle\right), & q_{s}=q_{l}=0, \\
q_{l}^{2}\left\langle\tilde{x}_{l}^{2}\right\rangle, & q_{o}=q_{s}=0,
\end{array}\right.
\end{aligned}
$$

which is identical to the rms variance calculations, setting $\beta=1$ for photons. For large relative momenta $q \gg 2 K_{\perp}$, we find instead

$$
\begin{aligned}
& \sum_{i j} q_{i} q_{j}\left\langle\left(\tilde{x}_{i}-\beta_{i}(q) \tilde{t}\right)\left(\tilde{x}_{j}-\beta_{j}(q) \tilde{t}\right)\right\rangle \\
&=\left\{\begin{array}{cl}
q_{s}^{2}\left\langle\tilde{x}_{s}^{2}\right\rangle, & q_{o}=q_{l}=0, \\
q_{o}^{2}\left\langle\tilde{x}_{o}^{2}\right\rangle-4 K_{\perp}\left|q_{o}\right|\left\langle\tilde{x}_{o} \tilde{t}\right\rangle+4 K_{\perp}^{2}\left\langle\tilde{t}^{2}\right\rangle, & q_{s}=q_{l}=0, \\
q_{l}^{2}\left\langle\tilde{x}_{l}^{2}\right\rangle, & q_{o}=q_{s}=0 .
\end{array}\right.
\end{aligned}
$$

We see that in this second limit, factors of $\tilde{t}$ come without factors of $q_{o}$, but with factors of $K_{\perp}$ instead, and this changes the $q$ dependence of the correlator in the $q_{o}$ direction. We note that if we had considered photon pairs with nonzero pair rapidity, an analogous difference would have also appeared between the last lines in Eqs. (29) for $q_{o} \ll 2 K$ and (30) for $q \gg 2 K$, where $K=\sqrt{K_{\perp}^{2}+K_{l}^{2}}$.

Figure 3 assumes $K_{l}=0$, hence for a Gaussian source, $q_{o}^{2} R_{l}^{2}=q_{o}^{2}\left\langle\tilde{x}_{l}^{2}\right\rangle$ holds at all values of $q$; the change of slope of the longitudinal correlator seen in Fig. 3 thus reflects deviations of the emission function from a Gaussian form along the longitudinal direction. We will discuss these in more detail further below. The sideward HBT radius is always free from temporal factors, since $\beta_{s} \equiv 0$ by definition. The sideward correlator in Fig. 3 exhibits a perfectly constant slope, indicating that the hydrodynamic photon emission function is well described by a Gaussian in the $x_{s}$ direction.

At large relative momentum $q_{o}$ the first term in the middle line of Eq. (30) dominates, and the slope of $\ln (C-1)$ converges to $\left\langle\tilde{x}_{o}^{2}\right\rangle$, with no dependence on the emission duration left. This is interesting because it allows us to separate the geometric from the temporal structure of the source with a single accurate measurement of the correlator at a fixed value of $K_{\perp}$, by studying its different slopes at small and large $q_{o}$. (We are aware, of course, of the experimental challenges of measuring thermal photon correlations at very small values of the pair momentum $K_{\perp}$.) With massive hadron 
pairs, this is impossible; for them, Eq. (28) is always a good approximation (as long as the source is sufficiently Gaussian), so a separation of geometric and temporal contributions requires measurements for different pair velocities $\beta \sim K_{\perp}$, with the additional assumption that the values of $\left\langle\tilde{x}^{2}\right\rangle,\langle\tilde{x} \tilde{t}\rangle$, and $\left\langle\tilde{t}^{2}\right\rangle$ do not themselves exhibit strong $K_{\perp}$ dependence.

According to Eq. (30), at large $q_{o}$, the outward slice of the correlator looks like a Gaussian with slope $\left\langle\tilde{x}_{o}^{2}\right\rangle$ and reduced intercept $\lambda=\frac{1}{2} e^{-4 K_{\perp}^{2}\left\langle\tilde{t}^{2}\right\rangle}$. At small relative momentum, its slope is characterized by $\left.\left\langle\tilde{x}_{o}^{2}\right\rangle-2\left\langle\tilde{x}_{o} \tilde{t}\right\rangle+\left\langle\tilde{t}^{2}\right\rangle\right\rangle\left\langle\tilde{x}_{o}^{2}\right\rangle$; this is bigger than the large momentum slope since for the hydrodynamic source both $-2\left\langle\tilde{x}_{o} \tilde{t}\right\rangle$ and $\left\langle\tilde{t}^{2}\right\rangle$ are positive. Equations (29) and (30) thus explain qualitatively the change of slope along the outward direction seen in Fig. 3.

The dotted and double-dash-dotted lines in Fig. 3 explore to what extent they also do so quantitatively. We see that they do not: the dotted line computed from Eq. (29) for $q_{o} \ll 2 K$ is much too steep, whereas the double-dash-dotted line computed from Eq. (30) for $q \gg 2 K$, while having the correct slope, is much too low. The reason for this failure is subtle. In the limit $K_{\perp}=0$, our photon emission function is independent of space-time rapidity $\eta$ since we use a hydrodynamic model with longitudinal boost invariance. In this limit, the variances $\left\langle\tilde{z}^{2}\right\rangle$ and $\left\langle\tilde{t}^{2}\right\rangle$ are infinite. For small nonzero $K_{\perp}$, they are finite but very large. When this happens, the expression (9), together with Eq. (10), can no longer be used because it relies on a Taylor expansion of the correlator around $\boldsymbol{q}=0$, keeping only the first nontrivial term and reexponentiating the result. This works only for Gaussian source, but for $K_{\perp} \rightarrow 0$ our source becomes very non-Gaussian along the $\eta$ direction, leading to a failure of this procedure in the terms containing $\left\langle\tilde{z}^{2}\right\rangle$ and $\left\langle\tilde{t}^{2}\right\rangle$.

The Gaussian approximation continues to work for the first term $\sim\left\langle\tilde{x}_{o}^{2}\right\rangle$ in the middle Eq. (30), which is why the doubledash-dotted line in Fig. 3 correctly reproduces the slope of the outward correlator slice (black solid line). In fact, this slope is the same as for the sideward correlator slice (red dashed line), indicating $\left\langle\tilde{x}_{o}^{2}\right\rangle=\left\langle\tilde{x}_{s}^{2}\right\rangle$ as should be the case for the central collisions studied in Fig. 3, owing to the azimuthal symmetry around the beam axis.

As a check for the above analysis, we performed a calculation of the correlator with a modified source in which we multiplied the photon emission function from AZHYDRO by hand with a Gaussian cutoff in the $\eta$ direction, $S(x, K) \mapsto$ $S(x, K) \times e^{-\eta^{2}}$. This simulates a fireball with finite rapidity width $\Delta \eta=1 / \sqrt{2}$. Now the longitudinal and temporal variances $\left\langle\tilde{z}^{2}\right\rangle$ and $\left\langle\tilde{t}^{2}\right\rangle$ remain finite even for $K_{\perp} \rightarrow 0$, and the non-Gaussian features of the underlying boost invariant hydrodynamic emission function (which set in at larger $\eta$ values) are suppressed. Figure 4 shows that in this case, the slopes and magnitudes of the outward correlator slice are very well reproduced by Eqs. (29) and (30). The problem in Fig. 3 is thus entirely due to the boost invariance of our hydrodynamic model.

As a corollary, we note that in contrast to hadrons whose rest mass always restricts their longitudinal homogeneity regions to small subvolumes whose longitudinal extent is controlled by the inverse of the longitudinal expansion rate [21,22], lowmomentum photons explore the full longitudinal size of the

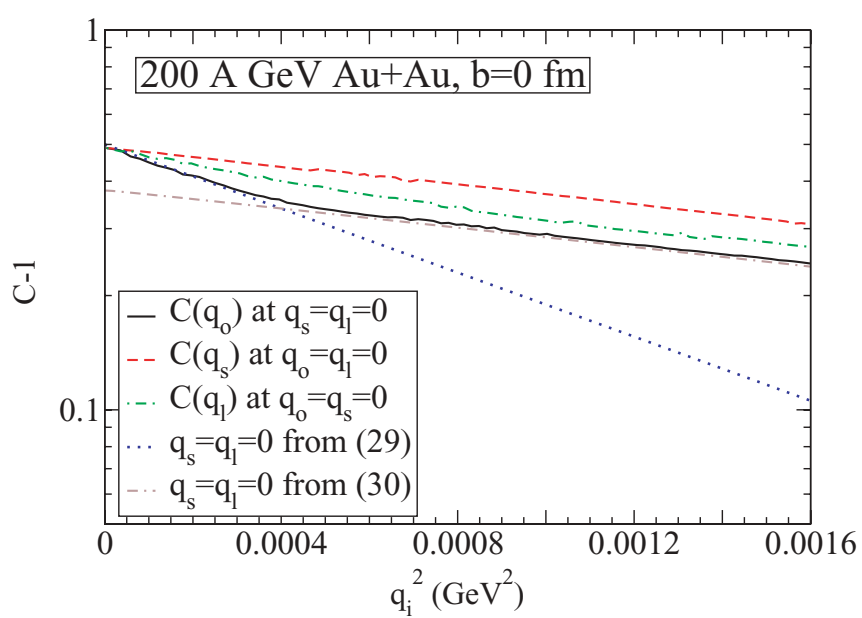

FIG. 4. (Color online) Same as Fig. 3, but for a modified emission function that has been multiplied by hand with a Gaussian cutoff factor $e^{-\eta^{2}}$ (see text for discussion).

expanding fireball. They can thus tell the difference between a longitudinally finite and an infinite boost invariant source. Quantitatively reliable predictions of two-photon correlations for pairs with small transverse momentum $K_{\perp}$ must therefore be based on realistic 3D evolution models that do not assume a boost invariant density distribution.

\section{Smoothness approximation}

In addition to the small $K_{\perp}$ breakdown of the on-shell approximation, the smoothness approximation also creates deviations which must be examined. The smoothness approximation is accurate as long as the curvature logarithm of the single-particle spectra is small [64]. However, with the emission rates used here, the photon spectra grow very rapidly at low momentum (see Fig. 1 of Ref. [51]), suggesting that in this region the smoothness approximation may also break down.

To explore the validity of the smoothness approximation, we write the correlator (3) as

$$
C(\boldsymbol{q}, \boldsymbol{K})=1 \pm \frac{1}{g_{s}} D(\boldsymbol{q}, \boldsymbol{K})\left|\frac{\int d^{4} x S(x, K) e^{i q \cdot x}}{\int d^{4} x S(x, K)}\right|^{2}
$$

where the last factor invokes the smoothness approximation (5), while deviations from this approximation are captured by the "correction factor"

$$
D(\boldsymbol{q}, \boldsymbol{K})=\frac{\left|\int d^{4} x S(x, \boldsymbol{K})\right|^{2}}{\int d^{4} x S\left(x, \boldsymbol{K}+\frac{\boldsymbol{q}}{2}\right) \int d^{4} x S\left(x, \boldsymbol{K}-\frac{\boldsymbol{q}}{2}\right)} .
$$

When the smoothness approximation is valid, $D \approx 1$.

We note that the correction factor $D$ is much harder to evaluate than the "smoothed" correlator (5), which can be efficiently computed by Monte Carlo integration with $S$ as the weight function. For this reason, we usually evaluated the $3 \mathrm{D}$ correlator $C(\boldsymbol{q}, \boldsymbol{K})$ in the smoothness approximation and used this as the basis for our 3D Gaussian fits of the HBT radii. Only for very small $K_{\perp}$ we also computed the correction factor $D$ 


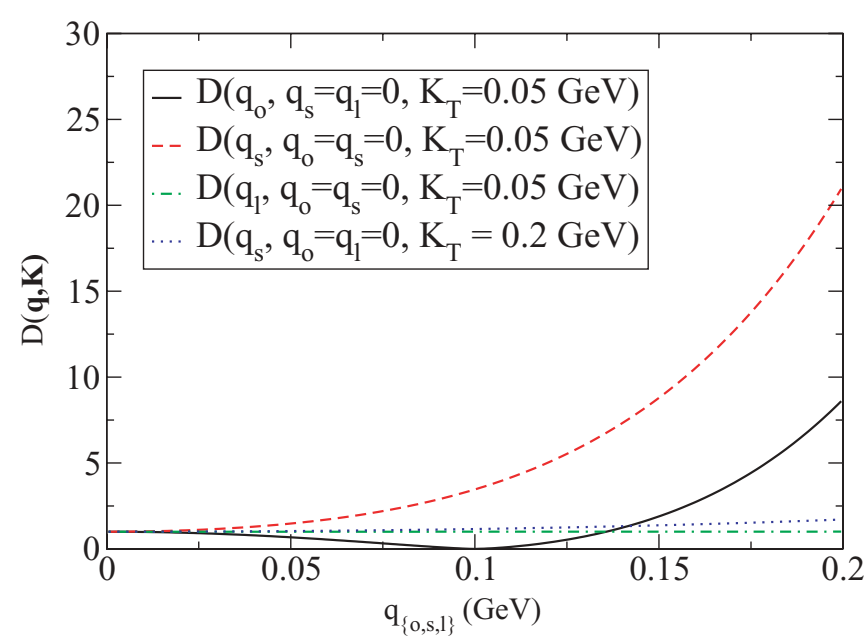

FIG. 5. (Color online) Smoothness correction factor [Eq. (32)] along the outward $\left(q_{o}\right.$, black solid), sideward $\left(q_{s}\right.$, red dashed), and longitudinal ( $q_{l}$, green dot-dashed) relative momentum axes, for central $\mathrm{Au}+\mathrm{Au}$ collisions at $200 \mathrm{~A} \mathrm{GeV}$ and for pair momentum $K_{\perp}=0.05 \mathrm{GeV}$. For comparison, the correction factor along the sideward direction $q_{s}$ is also shown for pairs with larger transverse momentum $K_{\perp}=0.2 \mathrm{GeV}$ (blue dotted line).

(see Fig. 8 below). In the present subsection, we check the validity of the smoothness approximation by computing the correction factor $D(\boldsymbol{q}, \boldsymbol{K})$ only along the $q_{o}, q_{s}$, and $q_{l}$ axes.

Figure 5 shows the smoothness correction factor $D(\boldsymbol{q}, \boldsymbol{K})$ as a function of relative momentum for photon pairs with $K_{\perp}=0.05 \mathrm{GeV}$. As the relative momentum increases, the ratio $D$ begins to deviate from unity for both the outward (solid black) and sideward (dashed red) slices. Along the $q_{l}$ direction (green dash-dotted), one sees almost no violation of the smoothness approximation. The characteristic momentum that sets the scale for violations of the smoothness approximation is again $\boldsymbol{q}=2 \boldsymbol{K}$, i.e., the same scale that characterizes the breakdown of the on-shell approximation. Because of the infinite longitudinal size of our boost invariant hydrodynamical source, the single-photon spectrum diverges at zero photon momentum; as a result, the denominator of the ratio $D$ in Eq. (32) goes to infinity along the outward direction when $q_{o}=2 K_{\perp}$, generating a zero of $D\left(q_{o}, K_{\perp}\right)$ at this point. No such zero occurs in the sideward and longitudinal directions, where $\boldsymbol{K} \pm \frac{\boldsymbol{q}}{2}$ never vanishes.

As we will see below (see Fig. 7), the effects of the smoothness correction factor on the 1D correlator slices are largest in the $q_{s}$ direction. They matter only for small $K_{\perp} \lesssim 1 / R_{\text {source }}$. To illustrate this, we show as the blue dotted line in Fig. 5 the correction factor $D$ along the $q_{s}$ direction for pairs with $K_{\perp}=0.2 \mathrm{GeV}$ (which is larger than the inverse source radius in central $\mathrm{Au}+\mathrm{Au}$ collisions). In this case, the deviations from unity are negligible over the entire range where $C(\boldsymbol{q}, \boldsymbol{K})$ deviates from unity.

\section{Non-Gaussian behavior along the longitudinal direction}

The non-Gaussian character of the longitudinal slice of the two-photon correlator in Fig. 3 deserves some further discussion. It appears to be much stronger than previously observed for pions. As we will see below, a 1D Gaussian fit to the longitudinal correlation function leads to a $\sim 20 \%$ reduction of intercept parameter $\lambda_{l}$, whereas the analogous reduction for the two-pion correlator is only about 5\% [38]. This suggests that the non-Gaussian effects may be to some extent controlled by the rest mass of the emitted particles.

To explore this issue, let us consider the following simple model for an expanding thermalized Gaussian source [22]:

$$
\begin{aligned}
S(x, p)= & K m_{\perp} \cosh (\eta) \exp \left(-\frac{p_{\mu} u^{\mu}}{T}\right) \\
& \times \exp \left(-\frac{\boldsymbol{r}^{2}}{2 \Delta \boldsymbol{r}}-\frac{\left(\tau-\tau_{0}\right)^{2}}{2(\Delta \tau)^{2}}\right) .
\end{aligned}
$$

The constant $K$ contains factors such as particle spin, fugacity, and the emission duration $\Delta \tau$. The emission function (33) has been studied extensively (see Ref. [22] for a review). With our hydrodynamical model, it shares the exact longitudinal boost invariance. By expanding the Boltzmann factor around $\eta=0$,

$$
\frac{p_{\mu} u^{\mu}}{T} \approx \frac{m_{\perp}}{T}\left(1+\frac{\eta^{2}}{2}+\cdots\right),
$$

and evaluating the emission function integrals for the rms variances in Eq. (10) by saddle-point integration, one finds $[22,67]$

$$
R_{l}^{2} \approx \tau_{0}^{2} \frac{T}{m_{\perp}}
$$

where $\tau_{0}$ is the mean freeze-out time. This result suggests $T / m_{\perp}$ scaling for the longitudinal relative momentum $\left(q_{l}\right)$ dependence of the two-particle correlation function. To isolate rest mass effects on the correlation function, we should study it as a function of the scaled momentum $q_{l} \sqrt{T / m_{\perp}}$.

Figure 6 shows longitudinal slices of the two-particle correlation function for spinless bosons of varying mass ("pseudopions"), computed from the hydrodynamic emission function on the hadron freeze-out surface for central $\mathrm{Au}+\mathrm{Au}$ collisions at RHIC energies. The correlation functions are plotted logarithmically against the scaled variable $q_{l}^{2} \frac{T}{m_{\perp}}$; the nonlinear decrease of the correlators in this representation exposes their non-Gaussian character. At large $q_{l}$ the correlation functions are seen to drop much more slowly than expected from their (common) asymptotic slope at $q_{l}=0$. These deviations from a Gaussian shape are strongest for low-mass particles and disappear for heavy particles. At infinite hadron mass, the correlator approaches a Gaussian function with a slope that agrees with the rest mass independent limiting slope at $q_{l}=0$.

Figure 6 explains why we see stronger non-Gaussian effects in the longitudinal correlator for massless photons than for pions. Spot checks have shown that the non-Gaussian aspects are further enhanced in volume emission (as is the case for photons) relative to Cooper-Frye surface emission (as simulated in Fig. 6 for pseudopions). Since computing the volume emission function for photons is numerically more demanding than the surface emission function for pions, we do not present a systematic study of rest mass effects on volume emission. 


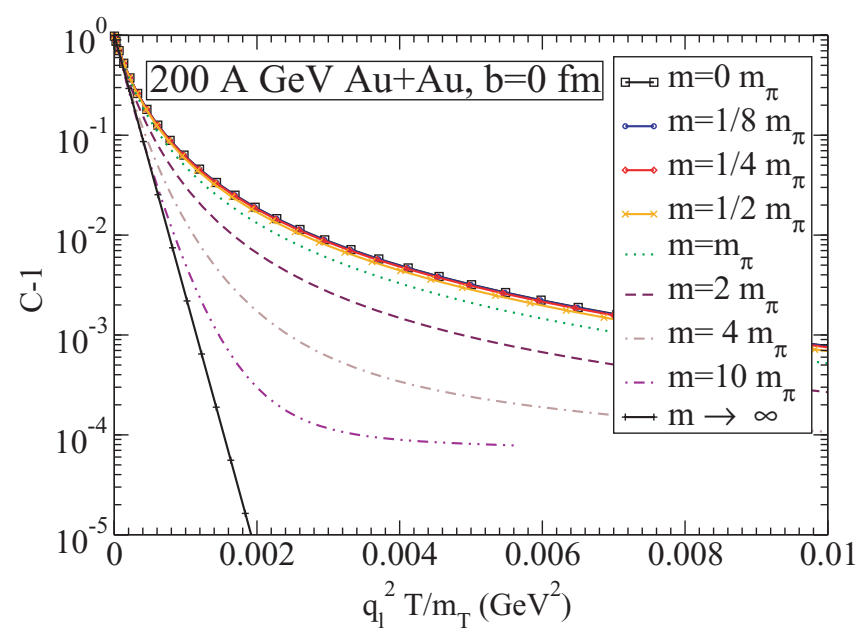

FIG. 6. (Color online) Longitudinal slices of the two-particle correlation function for spinless bosons ("pseudopions") in the limit of zero pair momentum, for different masses as given in the legend. The correlation functions were computed from the hydrodynamic pion emission function on the hadronic Cooper-Frye freeze-out surface, for $200 \mathrm{~A} \mathrm{GeV} \mathrm{Au}+\mathrm{Au}$ collisions at zero impact parameter, by varying the pion rest mass. They are plotted logarithmically against the scaled variable $q_{l}^{2} \frac{T}{m_{\perp}}$. See text for discussion.

\section{B. Photon HBT radii}

In the remainder of this work, we will concentrate on the transverse HBT radius parameters for thermal photons. But since the 3D correlation function is non-Gaussian along the longitudinal direction, this will in general contaminate the transverse HBT radii extracted from a 3D Gaussian fit even if the correlator is Gaussian in the transverse directions [38]. We deal with this problem as follows.

Figure 7 shows 1D slices of the 3D photon correlation function $C(\boldsymbol{q})-1$ along the $q_{o}, q_{s}$, and $q_{l}$ directions, together with 1D Gaussian fits using a 1D analog of the Gaussian fitting algorithm described in Sec. II C. The fits (solid lines) were done in the range $0<q_{i}<0.2 \mathrm{GeV} / c$. One notes the bad quality of this fit for the longitudinal slice [Fig. 7(d)], arising from the strongly non-Gaussian shape of the correlator in this direction, and the associated significantly reduced $(\sim 20 \%)$ intercept parameter. First principles tell us (and Fig. 6 confirms) that the correlator must be Gaussian near $q_{l}=0$. The dashed line in Fig. 7 shows that by restricting the $q_{l}$ fit range to $q_{l}<0.02 \mathrm{GeV} / c$ (i.e., a 10 times smaller window), we can largely cut out the non-Gaussian effects and obtain a fit with a reasonable intercept parameter close to 0.5 which is not in conflict with the similar intercept parameters preferred by the 1D Gaussian fits in $q_{s}$ and $q_{o}$ directions.

The dot-dashed lines show slices of the thermal photon correlation function of Eq. (3), which is obtained by multiplying the numerically computed curves in Fig. 7(a), which use the smoothness approximation Eq. (5), by the correction factor Eq. (32). Although both the outward and sideward slices are highly sensitive to deviations from the smoothed correlator at low relative momentum, the characteristic momentum $\boldsymbol{q}=2 \boldsymbol{K}$ is sufficiently in the tail of the correlator such that only the sideward slice exhibits these deviations. The deviations along $q_{o}$ only drive the correlator to zero faster and only in the tail when the correlator is sufficiently small.

In the following, we will therefore show transverse HBT radii that have been obtained from 3D Gaussian fits to the numerically calculated photon correlation functions $C(\boldsymbol{q})$ using a restricted $q_{l}$ fit range $\left|q_{l}\right|<0.02 \mathrm{GeV}$ in the longitudinal direction but including in the transverse directions the full range of $q_{o}, q_{s}$ values where $C$ deviates significantly from unity. This procedure is necessary if one wants to obtain meaningful values for the azimuthal oscillation amplitudes of the transverse HBT radii in noncentral collisions (see Sec. IV D2). If these are extracted from 3D fits with unrestricted $q_{l}$ range, the non-Gaussian features in $q_{l}$ lead to azimuthally oscillating intercept parameters $\lambda(\Phi)$ which contaminate and unphysically distort the azimuthal oscillations of the HBT radii in ways that do not reflect the azimuthal dependence of the size and shape of the emission function.

From the 3D Gaussian fits with restricted $q_{l}$ range, we also extract longitudinal HBT radii. Obviously, these reflect the curvature of the longitudinal correlator near $q_{l}=0$ and do not necessarily provide an accurate description of its longitudinal

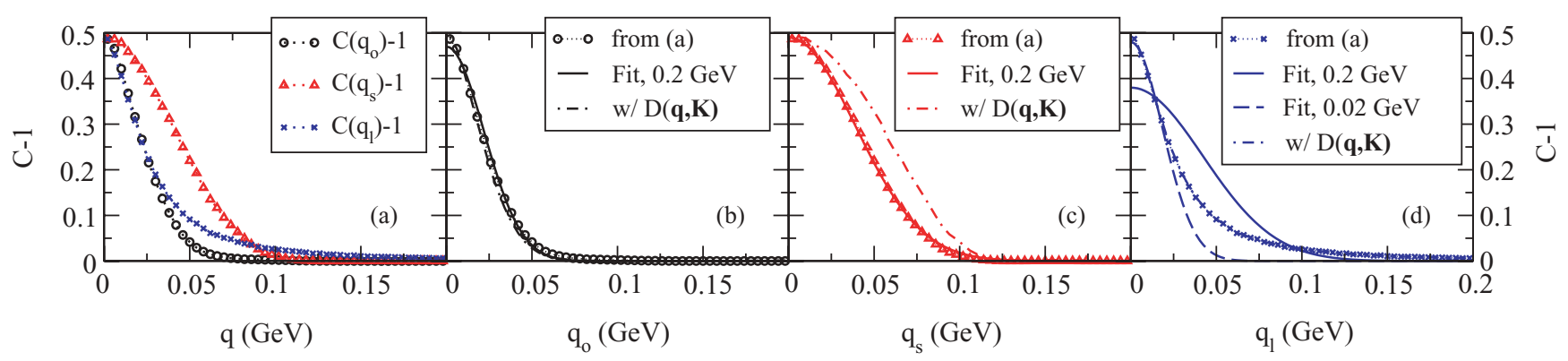

FIG. 7. (Color online) Slices of the photon correlation function $C(\boldsymbol{q})-1$ for central $\mathrm{Au}+\mathrm{Au}$ collisions at $\sqrt{s_{N N}}=200 \mathrm{GeV}$, for photon pairs with $K_{\perp}=0.05 \mathrm{GeV}$. Figure (a) shows slices of the numerically calculated 3D hydrodynamic correlation function along the $q_{o}$ (circles), $q_{s}$ (squares), and $q_{l}$ (triangles) directions. Note the strongly non-Gaussian shape of the $q_{l}$ slice. The other three panels (b)-(d) show the individual slices superimposed with $1 \mathrm{D}$ Gaussian fits, using a fit range $q_{i}<q_{\max }$ with $q_{\max }=0.2 \mathrm{GeV} / c$ (solid lines) and (in the longitudinal direction only) $q_{\max }=0.02 \mathrm{GeV} / c$ (dashed line). The dot-dashed line is the full correlation function taking into account the correction factor $D$ in Eq. (32) (see text for discussion). 
width. The latter is more accurately given by 1D Gaussian fits along $q_{l}$, and we will therefore show these 1D $R_{l}$ values for comparison.

\section{Central collisions}

Figure 8 (b) shows photon HBT radii from the hydrodynamic model for central $200 \mathrm{~A} \mathrm{GeV} \mathrm{Au}+\mathrm{Au}$ collisions as a function of photon pair momentum $K_{T}$, obtained from Gaussian fits to the numerically computed correlation function as described above. In outward and sideward directions, the 1D and 3D HBT radii are almost identical, except at small $K_{\perp}$, reflecting an approximately Gaussian shape of the correlator in transverse directions. As discussed in Sec. IV A, the outward two-photon correlator develops non-Gaussian features at small $K_{\perp}$, and the strong non-Gaussian features in the longitudinal direction (visible in the large discrepancy between the 1D and 3D longitudinal fit radii $R_{l}$ ) get also worse at small $K_{\perp}$.
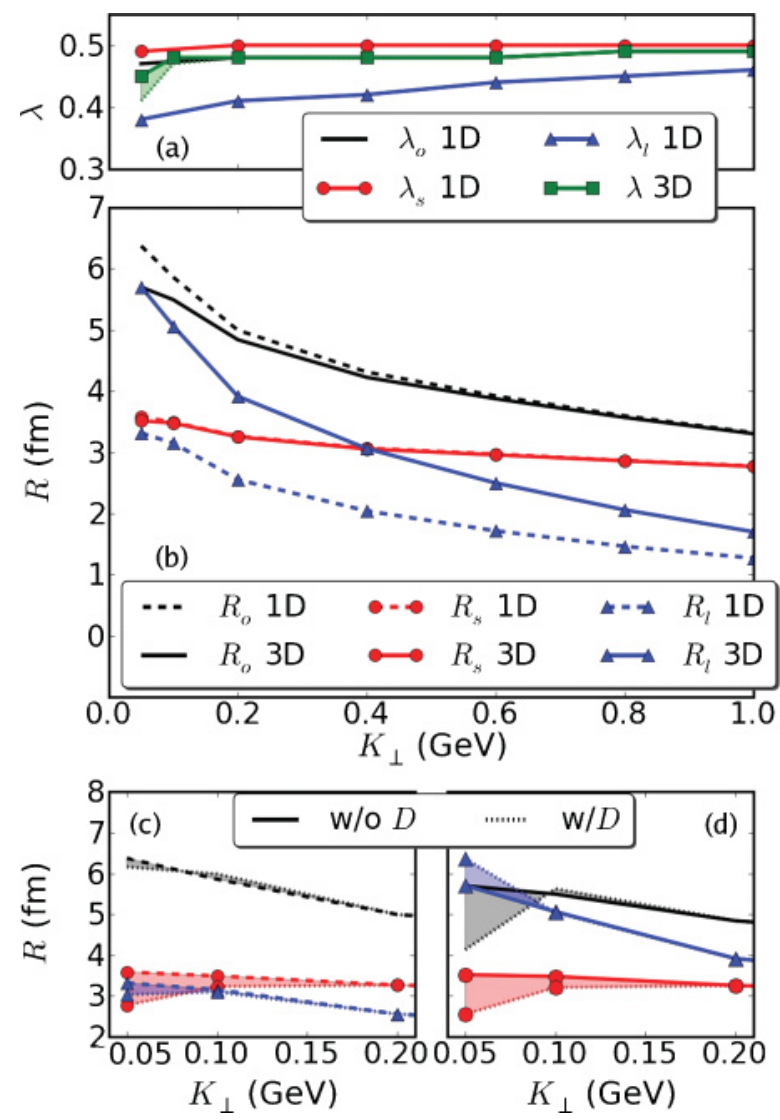

FIG. 8. (Color online) $K_{\perp}$ dependence of correlation strength parameter $\lambda$ (a) and HBT radii (b)-(d) from 1D- and 3D Gaussian fits of the hydrodynamic two-photon correlation function for central $\mathrm{Au}+\mathrm{Au}$ collision at RHIC energies. For the 3D fits, we used $q_{o, s}^{\max }=0.2 \mathrm{GeV}$ and $q_{l}^{\max }=0.02 \mathrm{GeV}$; the $1 \mathrm{D}$ longitudinal fits were done with $q_{l}^{\max }=0.2 \mathrm{GeV}$. (b) HBT radii from 1D and 3D Gaussian fits to the smoothness-approximated correlator (5). (c) Low- $K_{\perp}$ blowup of the 1D fit radii from (b) (dashed lines), compared with 1D fit radii for the smoothness-corrected correlator (31) (dotted lines). (d) Low- $K_{\perp}$ blowup of the 3D fit radii from (b) (solid lines), compared with 3D fit radii for the smoothness-corrected correlator (31) (dotted lines).
Both effects drive the common intercept parameter $\lambda$ for the 3D fit [squares in Fig. 8(a)] below 0.5, especially at low $K_{\perp}$, in spite of the restriction of the longitudinal fit range to $q_{l}<q_{l}^{\max }=0.02 \mathrm{GeV}$. For the 1D longitudinal fit which uses a 10 times larger longitudinal fit range, $\lambda$ shows a nearly $25 \%$ deviation from the expected value of 0.5 as $K_{\perp} \rightarrow 0$. This is our motivation for restricting the longitudinal fit range in the 3D fits, in order to minimize contamination of the transverse radii extracted from the $3 \mathrm{D}$ fit that stems from a reduction of the common intercept parameter exclusively driven by strongly non-Gaussian features along the longitudinal direction.

The dotted curves in Figs. 8(c) (1D radii) and 8(d) (3D radii) show the changes to the Gaussian fit radii when the smoothness correction factor (32) is applied to the correlator, as shown in Eq. (31). For the 1D fit radii, the smoothness correction factor has almost no effect along the outward and longitudinal directions; in the sideward direction, the broadening of the correlator by the smoothness correction factor, shown as the dash-dotted line in Fig. 7(c), leads to a $25 \%$ decrease of the 1D sideward radius $R_{s}$ at $K_{\perp}=0.05 \mathrm{GeV}$.

The 3D fits, however, show large smoothness correction effects for all three extracted radii at small $K_{\perp}$ where the factor $D$ in Eq. (32) introduces non-Gaussian deformations also in the sideward and outward directions (see Fig. 5). At $K_{\perp}=$ $0.05 \mathrm{GeV}$, these, together with the inherent non-Gaussian structure in the $q_{l}$ direction already at the smoothnessapproximated level, cause a reduction of the common 3D intercept parameter by almost $20 \%$ below its ideal value of $\frac{1}{2}$; this, in turn, further distorts the three Gaussian fit radii. These distortions happen only at very small pair momentum; already at $K_{\perp}=0.1 \mathrm{GeV}$ the smoothness correction effects are almost completely gone.

The most striking feature of Fig. 8(b) is the strong divergence between $R_{S}$ and $R_{o}$ as $K_{\perp} \rightarrow 0$. This is due to the temporal contributions (in particular the emission duration contribution) to $R_{o}^{2}$ which, for massless photons, are not suppressed by pair velocity factors in the limit $K_{\perp} \rightarrow 0$ as is the case for hadrons. As a result, for soft photon pairs, the outward HBT radius exceeds the sideward one by more than $50 \%$. We have checked that this effect disappears, i.e., $\lim _{K_{\perp} \rightarrow 0}\left(R_{o}-R_{s}\right)=0$ for the 3D Gaussian radii, if we give the photons a small nonzero mass.

In Fig. 9, we separate the photon emission function into two contributions, corresponding to radiation from the QGP and hadron gas (HG) phases. Not surprisingly, the HG HBT radii indicate a larger source for the hadronic phase than for the QGP phase, whose size is reflected in the QGP HBT radii. The measured photon HBT radii (QGP+HG) compromise between these values, reminding us that photons are emitted throughout the fireball evolution. At large $K_{\perp}$, the transverse HBT radii from the total source approach those from the QGP phase, reflecting the fact that at large $K_{\perp}$, QGP radiation dominates the single-photon yield. The transverse photon HBT radii from the HG phase are not too different in magnitude from the corresponding pion radii, except for the emission duration effects in $R_{o}$ at small $K_{\perp}$ for photons. The difference between $R_{o}$ and $R_{s}$ at small $K_{\perp}$ is smaller for the QGP than for the HG emission function, indicating shorter effective emission durations for QGP photons compared to hadronic photons. 


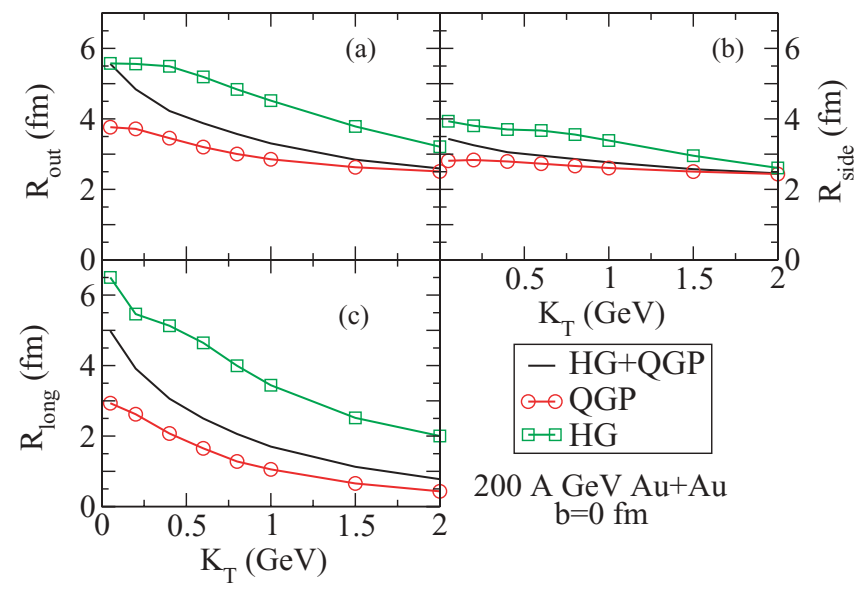

FIG. 9. (Color online) 3D Gaussian HBT radii as a function of transverse pair momentum for the photon emission functions describing radiation from the QGP and from the hadron gas stage (HG), and the total emission.

The longitudinal photon HBT radius from the HG phase is significantly smaller than the pionic one, but this is at least partially due to the stronger non-Gaussian effects in the longitudinal correlation function for photons and our restriction of the $q_{l}$ fit range in the $3 \mathrm{D}$ fits. The QGP photon radii are significantly smaller than the $\mathrm{HG}$ radii, especially for the longitudinal radius [59], reflecting the strong longitudinal expansion velocity gradient at early time $[22,67]$ when most of the QGP radiation is emitted [see also Eq. (35)], and acerbated by the strong non-Gaussian nature along the longitudinal direction. (We note that for photons, we find $R_{l}<R_{o}$ at all values of $K_{\perp}$, whereas the inverse relation $R_{o}<R_{l}$ holds for pions [38].)

\section{Non-central collisions}

\section{Photon emission functions}

The results from the previous subsection for central collisions have shown that the photon HBT radii can only be properly understood if one keeps in mind that photons are emitted from all stages of the expanding fireball. This is even more true for noncentral collisions and for the azimuthal oscillations of the HBT radii in this case. In this subsection, we show that at nonzero $K_{\perp} \sim 1 \mathrm{GeV}$, the photon emission functions exhibit qualitatively different characteristics from those we are familiar with from pions [71].

Figure 10 shows contour plots of the normalized emission function $S\left(x, y ; K_{\perp}\right)$, integrated over $z$ and $t$, for midrapidity photons at $K_{\perp}=0.05$ and $2 \mathrm{GeV} / c$. Since $K_{\perp}=0$ photons are emitted from essentially everywhere in the source at any time, but the space-time volume covered by the hadronic phase exceeds that of the QGP at RHIC energies [50], the contour lines in Figure 10(a) indicate the overall size and shape of the momentum-integrated photon emission function, with a stronger weight on the late evolution stages. The slight distortion of the contours toward the right (positive $x$ direction) reflects the nonzero pair momentum $K_{\perp}=0.05 \mathrm{GeV} / c$ in the $\Phi=0$ direction. Emission of such photons is dominated by

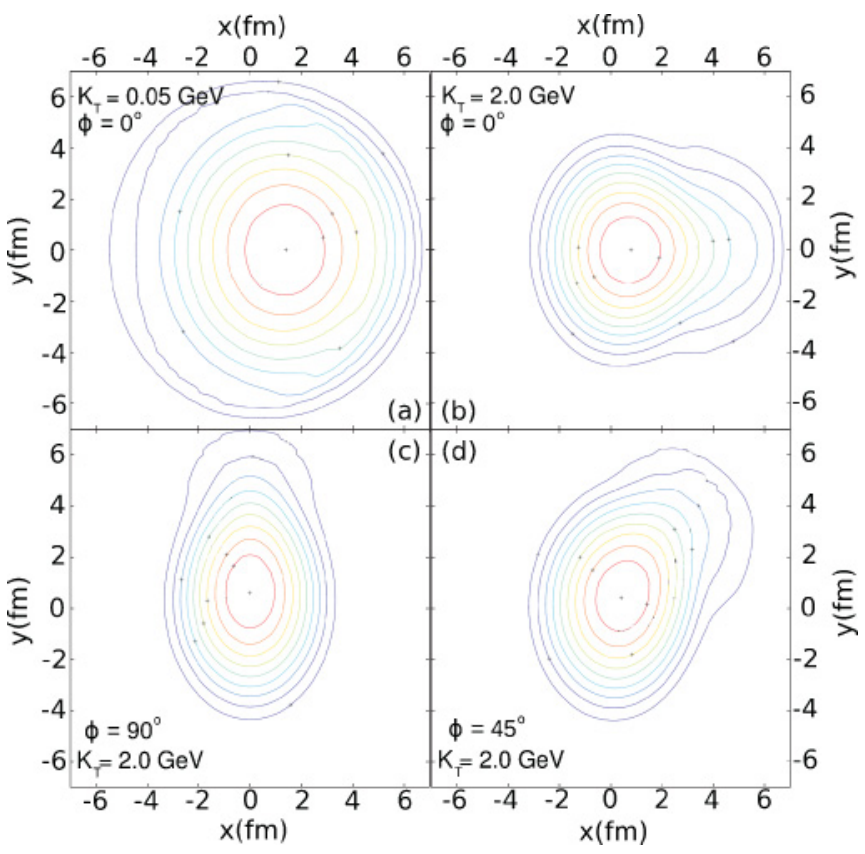

FIG. 10. (Color online) Photon emission function $S(x, y)$ at (a) $K_{\perp}=0.05 \mathrm{GeV} \approx 0, \Phi=0$, and (b)-(d) $K_{\perp}=2.0 \mathrm{GeV}$ for three emission directions, $\Phi=0^{\circ}, 45^{\circ}$, and $90^{\circ}$, for $200 \mathrm{~A} \mathrm{GeV} \mathrm{Au}+\mathrm{Au}$ collisions at $b=7 \mathrm{fm}$. The emission functions are normalized to unity at their maxima; contours are drawn in $10 \%$ intervals from this maximum value.

cells that flow hydrodynamically in the $+x$ direction with a flow velocity that has just the right magnitude to boost the peak of the Boltzmann distribution to this nonzero $K_{\perp}$ value. Because of the dominance of the $\mathrm{HG}$ phase for the emission of low- $K_{\perp}$ photons [50,51], this reflects the strong radial flow in the late hadronic stage of the fireball.

Photons with larger $K_{\perp}=2 \mathrm{GeV} / c$, on the other hand, are mostly emitted from the early QGP stage [50,51]. At this point, radial flow is weak, so we should naively expect the photon emission function to peak near the center of the fireball, with little distortion by radial flow. This is in strong contrast to pions that are only emitted at the end of the evolution from the hadronic freeze-out surface and thus always feel the full radial flow of the late hadronic fireball. For large $K_{\perp}$ values, hadronic emission functions are strongly peaked near the edge of the fireball, forming narrow crescent-shaped slivers that straddle the surface of the fireball [71], since it is in these regions that one finds the strongest radial flow which most efficiently boosts the local thermal distribution with temperature $T_{f}$ toward large $K_{\perp}$ values.

The three $K_{\perp}=2 \mathrm{GeV} / c$ panels [Figs. 10(b)-10(d)] show something quite different: For large $K_{\perp}$, the photon emission function peaks near the center of the fireball (at early times, as studies of its $t$ dependence have revealed), but it exhibits a "bulge" in the direction of the emitted photons that reflects a contribution from later times when the matter is in the hadronic phase and boosted by strong radial flow. Even though the hadronic phase radiates at much lower temperature $\left(\left\langle T_{\mathrm{HG}}\right\rangle \sim 150 \mathrm{MeV}\right.$, whereas $\left\langle T_{\mathrm{QGP}}\right\rangle$ is almost twice as high), which strongly suppresses its photon emission rate, the larger 
space-time volume covered by the HG phase partially compensates for that loss in rate and makes hadronic photon emission sufficiently competitive at $K_{\perp}=2 \mathrm{GeV} / c$ to give a visible flow-boosted contribution to the photon emission function. The hadronic component is not strong enough to collectively "squeeze" the photon emission function toward the edge of the fireball (as was the case for pions), but it distorts it visibly into the direction of the emitted photon pair. This parallels the study of photon elliptic flow which showed $[50,51]$ that even though at $K_{\perp}=2 \mathrm{GeV} / c$ the single-photon yield is dominated by QGP radiation, the stronger elliptic flow of the HG photons still controls the total photon $v_{2}$, and one must go to significantly larger transverse momenta before the elliptic flow of photons provides an uncontaminated view of the earliest QGP stage.

Were it not for the hadronic "bulge," the $K_{\perp}=2 \mathrm{GeV} / c$ emission functions shown in Fig. 10 would indeed provide a clean reflection of the original source eccentricity in noncentral collisions. The emission function in the Figs. 10(b)-10(d) (dominated by QGP radiation) is clearly more eccentric than the one in Fig. 10(a) (which is dominated by hadronic radiation). What Fig. 10 teaches us is that the full story is more complex than suggested by this naive view and that any interpretation of azimuthal oscillations of the photon HBT radii must properly account for the two-component structure of the photon emission function which is generated by the interplay between nonboosted QGP and flow-boosted hadronic photon emission, with relative weights that depend on $K_{\perp}$.

\section{Azimuthal oscillations of photon HBT radii}

Figure 11 shows the azimuthal oscillations of the transverse photon $\mathrm{HBT}$ radius parameters in semiperipheral $\mathrm{Au}+\mathrm{Au}$ collisions at RHIC. The HBT radii were extracted from a 3D Gaussian fit to the correlation function, with restricted longitudinal fit range $q_{l}<0.02 \mathrm{GeV} / c$ as explained above. Qualitatively, the features seen in Fig. 11 agree with those for pions shown in Fig. 2. On a more quantitative level, one observes important differences: the sideward radius is smaller for photons, reflecting preferred emission from the smaller, hotter fireball interior and earlier times, while the relative oscillation amplitude is larger, reflecting the stronger source eccentricity at early times. The $K_{\perp}$ dependence of the azimuthally averaged $R_{S}$ value is weaker for photons than for pions; the likely reason is weaker radial flow at earlier times when a large fraction of the photons are emitted. The $K_{\perp}$ dependence of $R_{o}$ is much stronger for photons than for pions, mostly because of the much larger photon $R_{o}$ radii at small $K_{\perp}$ resulting from the emission duration contribution, which at low $K_{\perp}$ is suppressed for pions but not for photons (see discussion in Sec. IV A).

Interestingly, the $R_{o s}^{2}$ cross term for photons oscillates with an amplitude that is almost independent of $K_{\perp}$ and even bigger at small $K_{\perp}$ than at large $K_{\perp}$. Figure 11 (c) exhibits almost pure $\sin (2 \Phi)$ oscillations for the $R_{o s}^{2}$. The relationship [69,71]

$$
\begin{aligned}
R_{o s}^{2}= & \cos (2 \Phi)\langle\tilde{x} \tilde{y}\rangle+\sin (2 \Phi) \frac{\left\langle\tilde{y}^{2}-\tilde{x}^{2}\right\rangle}{2} \\
& +\beta(q)(\langle\tilde{x} \tilde{t}\rangle \sin \Phi-\langle\tilde{y} \tilde{t}\rangle \cos \Phi)
\end{aligned}
$$

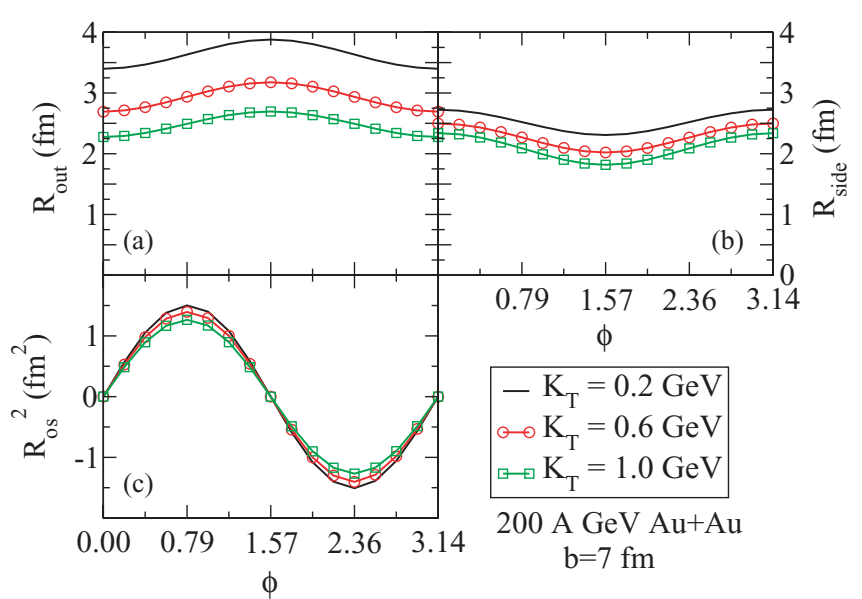

FIG. 11. (Color online) Azimuthal oscillations of the transverse photon HBT radius parameters (a) $R_{o}$, (b) $R_{s}$, and (c) $R_{o s}^{2}$, for photon pairs with $K_{\perp}=0.2,0.6$, and $1.0 \mathrm{GeV} / c$ from semiperipheral $200 A \mathrm{GeV} \mathrm{Au}+\mathrm{Au}$ collisions at $b=7 \mathrm{fm}$.

which holds for emission functions whose dependence on $x, y, t$ is Gaussian and should thus be reasonably accurate for our source, indicates that the larger $\sin (2 \Phi)$ oscillation amplitude for photons at low $K_{\perp}$ is caused by a larger source eccentricity for low-momentum photons than for lowmomentum pions. This is due to the significant weight of early photon emission even at zero transverse pair momentum.

For pions, it was found that $K_{\perp}=0$ pion pairs are emitted from the entire fireball, and that the normalized azimuthal oscillation amplitude of the sideward radius $R_{s}$ can thus be used to measure the spatial eccentricity of the fireball at pion freeze-out [79]. In the same spirit, we can try to extract the fireball eccentricity from the normalized azimuthal oscillations of the photon sideward radius at $K_{\perp}$. The sideward radius is the signature of choice, since it is a purely geometric observable, uncontaminated by temporal contributions [see Eqs. (29) and (30)]. The discussion above indicates, however, that photons even at $K_{\perp}=0$ are emitted more often from the hot fireball center at early times than from the cooler periphery at later times, so the normalized azimuthal $R_{S}$ oscillation for photons will measure the effective fireball eccentricity at earlier times than for pions. This is, of course, exactly what we hoped to obtain. The only unexpected aspect is that this would work even for $K_{\perp}=0$, where we did not anticipate early emission to play quite as important a role as we now see.

The contour plots in Fig. 10 show that the space-time character of photon emission differs from pions even more strongly at larger $K_{\perp}$, where photon emission is even more strongly concentrated at early times close to the fireball center, whereas pion emission is almost completely surface dominated and concentrated to a thin sliver near the fireball edge [71]. This makes a geometric interpretation of the normalized $R_{S}$ oscillation amplitude in terms of spatial eccentricity of the photon emission function possible even at larger $K_{\perp}$ values. By going to larger $K_{\perp}$, we can thus measure with photons the fireball eccentricity at even earlier times, by analyzing their normalized $R_{s}$ oscillation amplitudes as a function of $K_{\perp}$. 


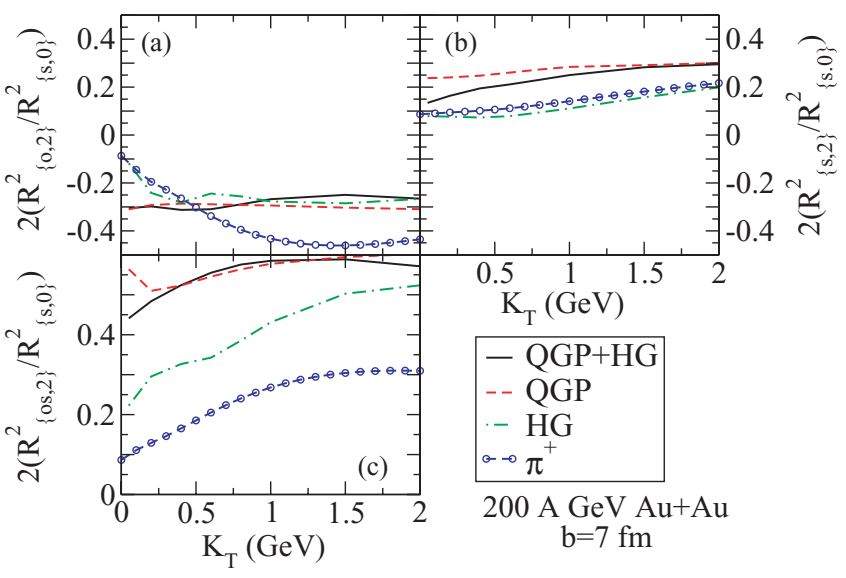

FIG. 12. (Color online) Normalized oscillation amplitudes of the photon (left column) and pion (right column) HBT radii from $200 A \mathrm{GeV} \mathrm{Au}+\mathrm{Au}$ collisions at $b=7 \mathrm{fm}$, as a function of transverse pair momentum.

(For pions, the straightforward geometric interpretation of this observable is lost for $K_{\perp} \gg 0$ [79].)

In Fig. 12, we show the normalized oscillation amplitudes [70] which, following Retiere and Lisa [79], can be expressed through the following normalized second-order azimuthal Fourier components of the HBT radius parameters $R_{i j}^{2}(\Phi)^{1}$ :

$$
\begin{aligned}
2 \frac{R_{(o, s), 2}^{2}}{R_{s, 0}^{2}} & =\frac{R_{(o, s)}^{2}(0)-R_{(o, s)}^{2}\left(\frac{\pi}{2}\right)}{R_{s}^{2}(0)+R_{s}^{2}\left(\frac{\pi}{2}\right)}, \\
2 \frac{R_{o s, 2}^{2}}{R_{s, 0}^{2}} & =\frac{R_{o s}^{2}\left(\frac{\pi}{4}\right)-R_{o s}^{2}\left(\frac{3 \pi}{4}\right)}{R_{s}^{2}(0)+R_{s}^{2}\left(\frac{\pi}{2}\right)} .
\end{aligned}
$$

The oscillation amplitudes for $R_{o}^{2}$ [Fig. 12(a)] and $R_{s}^{2}$ [Fig. 12(b)] differ mostly by an overall sign for both photons and pions; any additional differences in magnitude are due to azimuthal oscillations of the temporal contributions to $R_{o}^{2}[69,71]$. The latter are larger for pions than for photons. Fig. 12(c) shows that the azimuthal oscillations of the $R_{o s}^{2}$ cross term are much larger for photons than for pions (as already seen in Figs. 11 and 2), and that this difference is mostly due to the earlier emission of the photons (it is more pronounced for the photons from the QGP phase than from the hadron phase, but even in the HG phase this cross term oscillates more strongly for photons than for pions at large $K_{\perp}$ where HG photons are emitted earlier than pions).

The most important information that can be extracted from Fig. 12 comes from the oscillation amplitude of $R_{s}^{2}$. Figure 12(b) shows that $R_{s}^{2}$ oscillates more strongly for photons emitted from the QGP stage than from the hadron phase. This reflects the larger eccentricity of the fireball at earlier times. The oscillation amplitude for the total photon emission function interpolates between the QGP and $\mathrm{HG}$ limits: for $K_{\perp} \gtrsim 2 \mathrm{GeV} / c$ it coincides with the QGP curve (indicating complete QGP dominance for $K_{\perp}>2 \mathrm{GeV} / c$ ), but

\footnotetext{
${ }^{1}$ We note that in Eq. (1) of Ref. [73] we inadvertently forgot the factor 2 on the left-hand side of Eqs. (38).
}

even at $K_{\perp}=0$ the QGP contribution still plays a significant role. Retiere and Lisa [79] showed for pions that one can extract the source eccentricity at freeze-out from the relation [see also Eq. (2) in Ref. [71]]

$$
\epsilon_{x}=2 R_{s, 2}^{2} / R_{s, 0}^{2}
$$

in the limit $K_{\perp} \rightarrow 0$. In Ref. [74], this procedure was successfully applied to RHIC data. We have argued above that for photons, a geometric interpretation of the right-hand side in Eq. (38) should remain possible at nonzero $K_{\perp}$. If this is true, we can follow the fireball eccentricity backward in time by following the black solid line in Fig. 12(a) from low to high $K_{\perp}: K_{\perp}=0$ would represent a time somewhere around $T_{c}$, whereas for $K_{\perp}>2 \mathrm{GeV} / c$, we would probe times close to thermalization of the QGP. Of course, fixed $K_{\perp}$ values cannot be mapped one-to-one to sharply defined emission times-the intention of our argument is to point out a qualitative and novel tendency that becomes accessible with photon interferometry. The analogous procedure for pion HBT oscillations has no similar meaningful geometric interpretation.

\section{CONCLUSIONS}

In this paper, we presented a first comprehensive study of two-photon correlations and the azimuthal oscillations of the photon HBT radii in noncentral heavy-ion collisions. We based our investigation on the hydrodynamic photon emission function for $\mathrm{Au}+\mathrm{Au}$ collisions at $\sqrt{s}=200 \mathrm{~A} \mathrm{GeV}$ with impact parameter $b=7 \mathrm{fm}$ as a model system. By comparing photon and pion HBT correlations, we were able to identify several key differences, both in the formalism and in the numerical results, that make azimuthally sensitive photon interferometry an exciting prospect for future experimental studies.

The two most important insights from this study are that (i) that since real photons are massless, a qualitative change of the shape of the two-particle correlator $C(\boldsymbol{q})$ for soft photon pairs with $K \lesssim 1 / R_{\text {fireball }}$ develops which, if it can be measured, will allow us to separate the spatial and temporal aspects of photon emission in a model-independent fashion, and (ii) at all transverse momenta within the range of validity of the hydrodynamic model, photon emission is essentially volume dominated, with a strong QGP component from early times close to the fireball center and rather weak distortions from a secondary flow-boosted hadronic emission component that extends more toward the edge of the fireball in the emission direction. The first observation requires measuring two-photon correlations at very small pair momentum, which will be very difficult; it also requires a careful shape analysis of the two-photon correlator in relative momentum, since both the on-shell and smoothness approximations break down in this $K_{\perp}$ range, and the correlator becomes strongly non-Gaussian. The second feature is more useful in practice, since it works over a large range of pair momenta; it allows the use of photon HBT radii for measuring the size and shape of the fireball at early times, before hadronization and hadronic freeze-out, and to map out the time evolution of its spatial eccentricity, by studying the normalized azimuthal oscillation amplitude 
of the sideward radius as a function of transverse photon pair momentum. This realizes a long-held dream for using two-photon correlations as a microscope for measuring the early fireball geometry.

\section{ACKNOWLEDGMENTS}

We gratefully acknowledge stimulating discussions with Mike Lisa who informed us that he, in unpublished work with
Giuseppe Verde, had previously seen evidence for breakdown of the on-shell and smoothness approximations for pion pairs at very small transverse pair momentum. We also thank Joe Kapusta for helpful discussions and constructive comments on an earlier version of this manuscript. Finally we thank Rupa Chatterjee and Dinesh Srivastava for some very informative discussions. The work reported here is in part based on the $\mathrm{Ph}$.D. thesis of E.F. written at the Ohio State University. It was supported by the US Department of Energy under Grant Nos. DE-FG02-01ER41190 and DE-FG02-87ER40328.
[1] P. F. Kolb, J. Sollfrank, and U. Heinz, Phys. Lett. B459, 667 (1999).

[2] P. F. Kolb, J. Sollfrank, and U. Heinz, Phys. Rev. C 62, 054909 (2000).

[3] D. Teaney, J. Lauret, and E. V. Shuryak, Phys. Rev. Lett. 86, 4783 (2001).

[4] D. Teaney, J. Lauret, and E. V. Shuryak, arXiv:nucl-th/0110037.

[5] P. Huovinen, P. F. Kolb, U. Heinz, P. V. Ruuskanen, and S. A. Voloshin, Phys. Lett. B503, 58 (2001).

[6] T. Hirano and K. Tsuda, Phys. Rev. C 66, 054905 (2002).

[7] P. F. Kolb and R. Rapp, Phys. Rev. C 67, 044903 (2003).

[8] K. H. Ackermann et al. (STAR Collaboration), Phys. Rev. Lett. 86, 402 (2001).

[9] C. Adler et al. (STAR Collaboration), Phys. Rev. Lett. 87, 182301 (2001).

[10] C. Adler et al. (STAR Collaboration), Phys. Rev. Lett. 87, 262302 (2001).

[11] K. Adcox et al. (PHENIX Collaboration), Phys. Rev. Lett. 88, 242301 (2002).

[12] J. Adams et al. (STAR Collaboration), Nucl. Phys. A757, 102 (2005).

[13] K. Adcox et al. (PHENIX Collaboration), Nucl. Phys. A757, 184 (2005).

[14] P. Huovinen, in Quark-Gluon Plasma 3, edited by R. Hwa and X.-N. Wang (World Scientific, Singapore, 2004), p. 600.

[15] P. F. Kolb and U. Heinz, in Quark-Gluon Plasma 3, edited by R. Hwa and X.-N. Wang (World Scientific, Singapore, 2004), p. 634.

[16] M. A. Lisa, S. Pratt, R. Soltz, and U. Wiedemann, Annu. Rev. Nucl. Part. Sci. 55, 357 (2005).

[17] D. H. Boal, C. K. Gelbke, and B. K. Jennings, Rev. Mod. Phys. 62, 553 (1990).

[18] U. Heinz, Nucl. Phys. A610, 264c (1996).

[19] U. Heinz, in Correlations and Clustering Phenomena in Subatomic Physics, NATO ASI Series B, Physics, Vol. 359, edited by M. N. Harakeh, J. H. Koch, and O. Scholten (Plenum Press, 1997), p. 137.

[20] R. Hanbury Brown and R. Q. Twiss, Nature 178, 1046 (1956).

[21] U. Heinz and B. V. Jacak, Annu. Rev. Nucl. Part. Sci. 49, 529 (1999).

[22] U. A. Wiedemann and U. Heinz, Phys. Rep. 319, 145 (1999).

[23] U. Heinz and P. F. Kolb, in Proceedings of the 18th Winter Workshop on Nuclear Dynamics, edited by R. Bellwied, J. Harris, and W. Bauer (EP Systema, Debrecen, Hungary, 2002), p. 205.

[24] S. Soff, S. A. Bass, and A. Dumitru, Phys. Rev. Lett. 86, 3981 (2001).

[25] O. Socolowski, F. Grassi, Y. Hama, and T. Kodama, Phys. Rev. Lett. 93, 182301 (2004).

[26] T. Renk, Phys. Rev. C 70, 021903(R) (2004).
[27] W. Broniowski, M. Chojnacki, W. Florkowski, and A. Kisiel, Phys. Rev. Lett. 101, 022301 (2008).

[28] D. Teaney, Phys. Rev. C 68, 034913 (2003).

[29] P. Romatschke, Eur. Phys. J. C 52, 203 (2007).

[30] S. Pratt and J. Vredevoogd, Phys. Rev. C 78, 054906 (2008).

[31] S. Pratt, Phys. Rev. Lett. 102, 232301 (2009).

[32] J. G. Cramer, G. A. Miller, J. M. S. Wu, and J.-H. Yoon, Phys. Rev. Lett. 94, 102302 (2005).

[33] S. Pratt, Phys. Rev. C 73, 024901 (2006).

[34] J. I. Kapusta and Y. Li, Phys. Rev. C 72, 064902 (2005).

[35] A. J. Kuhlman, Ph.D. thesis, The Ohio State University, 2007.

[36] W. Florkowski, M. Chojnacki, W. Broniowski, and A. Kisiel, Acta Phys. Polon. B 39, 1555 (2008).

[37] A. Kisiel, W. Broniowski, M. Chojnacki, and W. Florkowski, Phys. Rev. C 79, 014902 (2009).

[38] E. Frodermann, U. Heinz, and M. A. Lisa, Phys. Rev. C 73, 044908 (2006).

[39] U. Heinz and P. F. Kolb, Nucl. Phys. A702, 269 (2002).

[40] M. Gyulassy and L. McLerran, Nucl. Phys. A750, 30 (2005).

[41] P. Romatschke and U. Romatschke, Phys. Rev. Lett. 99, 172301 (2007).

[42] H. Song and U. Heinz, Phys. Lett. B658, 279 (2008).

[43] M. Luzum and P. Romatschke, Phys. Rev. C 78, 034915 (2008).

[44] H. Song and U. Heinz, J. Phys. G: Nucl. Part. Phys. 36, 064033 (2009).

[45] E. V. Shuryak, Phys. Lett. B78, 150 (1978).

[46] K. Kajantie and H. I. Miettinen, Z. Phys. C 9, 341 (1981).

[47] K. Kajantie, J. I. Kapusta, L. D. McLerran, and A. Mekjian, Phys. Rev. D 34, 2746 (1986).

[48] P. V. Ruuskanen, in Quark Gluon Plasma, edited by R. C. Hwa, Adv. Ser. Dir. High Energy Physics, Vol. 6 (World Scientific, 1990), p. 519.

[49] P. Ruuskanen, in Particle Production in Highly Excited Matter, NATO ASI Series B: Physics, Vol. 303, edited by H. Gutbrod and J. Rafelski (Plenum, New York, 1993), p. 593.

[50] R. Chatterjee, E. S. Frodermann, U. Heinz, and D. K. Srivastava, Phys. Rev. Lett. 96, 202302 (2006).

[51] U. Heinz, R. Chatterjee, E. S. Frodermann, C. Gale, and D. K. Srivastava, Nucl. Phys. A783, 379 (2007).

[52] R. Chatterjee and D. K. Srivastava, Phys. Rev. C 79, 021901 (2009)

[53] H. Song and U. Heinz, Phys. Rev. C 77, 064901 (2008).

[54] H. Song and U. Heinz, Phys. Rev. C 78, 024902 (2008).

[55] D. K. Srivastava and J. I. Kapusta, Phys. Lett. B307, 1 (1993).

[56] D. K. Srivastava and J. I. Kapusta, Phys. Rev. C 48, 1335 (1993). 
[57] D. K. Srivastava, Phys. Rev. D 49, 4523 (1994).

[58] D. K. Srivastava, Phys. Rev. C 71, 034905 (2005).

[59] S. A. Bass, B. Muller, and D. K. Srivastava, Phys. Rev. Lett. 93, 162301 (2004).

[60] M. Lisa, E. Frodermann, and U. Heinz, Braz. J. Phys. 37, 903 (2007).

[61] S. Turbide, C. Gale, E. Frodermann, and U. Heinz, Phys. Rev. C 77, 024909 (2008).

[62] C. Gale, S. Turbide, E. Frodermann, and U. Heinz, J. Phys. G 35, 104119 (2008).

[63] P. Braun-Munzinger, D. Magestro, K. Redlich, and J. Stachel, Phys. Lett. B518, 41 (2001).

[64] S. Chapman, P. Scotto, and U. Heinz, Acta Phys. Hung. New Series: Heavy Ion Physics 1, 1 (1995).

[65] S. Pratt, Phys. Rev. C 56, 1095 (1997).

[66] U. Heinz, in 2003 CERN-CLAF School of High-Energy Physics, edited by N. Ellis, CERN Yellow Report CERN-2006-001, p. 165, arXiv:hep-ph/0407360.

[67] A. N. Makhlin and Y. M. Sinyukov, Z. Phys. C 39, 69 (1988).
[68] U. A. Wiedemann, Phys. Rev. C 57, 266 (1998).

[69] M. A. Lisa, U. Heinz, and U. A. Wiedemann, Phys. Lett. B489, 287 (2000).

[70] U. Heinz, A. Hummel, M. A. Lisa, and U. A. Wiedemann, Phys. Rev. C 66, 044903 (2002).

[71] U. Heinz and P. F. Kolb, Phys. Lett. B542, 216 (2002).

[72] S. Chapman and U. Heinz, Phys. Lett. B340, 250 (1994).

[73] E. Frodermann, R. Chatterjee, and U. Heinz, J. Phys. G 34, 2249 (2007).

[74] J. Adams et al. (STAR Collaboration), Phys. Rev. Lett. 93, 012301 (2004).

[75] S. A. Bass, B. Muller, and D. K. Srivastava, Phys. Lett. B551, 277 (2003).

[76] C. Slotta and U. Heinz, Phys. Lett. B391, 469 (1997).

[77] B. Tomasik and U. W. Heinz, arXiv:nucl-th/9805016.

[78] U. A. Wiedemann and U. Heinz, Phys. Rev. C 56, 3265 (1997).

[79] F. Retiere and M. A. Lisa, Phys. Rev. C 70, 044907 (2004). 